\title{
The Cortical Representation of the Hand in Macaque and Human Area S-I: High Resolution Optical Imaging
}

\author{
Doron Shoham and Amiram Grinvald \\ Department of Neurobiology, Weizmann Institute of Science, Rehovot 76100, Israel
}

\begin{abstract}
High-resolution images of the somatotopic hand representation in macaque monkey primary somatosensory cortex (area S-I) were obtained by optical imaging based on intrinsic signals. To visualize somatotopic maps, we imaged optical responses to mild tactile stimulation of each individual fingertip. The activation evoked by stimulation of a single finger was strongest in a narrow transverse band $(\sim 1 \times 4 \mathrm{~mm})$ across the postcentral gyrus. As expected, a sequential organization of these bands was found. However, a significant overlap, especially for the activated areas of fingers 3-5, was found. Surprisingly, in addition to the finger-specific domains, we found that for each of the fingers, weak stimulation activated also a second "common patch" of cortex, located just medially to the representation of the finger. These results were confirmed by targeted multiunit and single-unit recordings guided by the optical maps. The maps remained very stable over many hours of recording. By
\end{abstract}

optimizing the imaging procedures, we were able to obtain the functional maps extremely rapidly (e.g., the map of five fingers in the macaque monkey could be obtained in as little as $5 \mathrm{~min}$ ). Furthermore, we describe the intraoperative optical imaging of the hand representation in the human brain during neurosurgery and then discuss the implications of the present results for the spatial resolution accomplishable by other neuroimaging techniques, relying on responses of the microcirculation to sensoryevoked electrical activity. This study demonstrates the feasibility of using high-resolution optical imaging to explore reliably short- and long-term plasticity of cortical representations, as well as for applications in the clinical setting.

Key words: monkey; macaque; somatosensory cortex; somatotopy; area S-I; area 1; functional architecture; cortical maps; optical imaging; intrinsic signals; f-MRI; PET; neurosurgery
Research over the past few decades has established that many sensory stimuli are represented in primary sensory cortices in the form of functional maps. Thus, cells with similar stimulus preferences are clustered in cortical columns, forming twodimensional maps of stimulus properties on the surface of the cortex (Mountcastle, 1957: Hubel and Wiesel, 1965). The pioneering intrinsic optical imaging explorations of S-I somatosensory cortex in squirrel monkeys (Tommerdahl et al., 1993, 1996a,b, 1998, 1999a,b) have addressed issues of the topography in S-I responses to cutaneous flutter, vibration, tapping, and skin heating. However, high-resolution imaging of the macaque monkey S-I has not yet been reported. The objective of this study was to explore the somatotopic representation of all five fingers together, which previous studies have not examined. The benefit of studying S-I in macaques lies in the well established advantage of this species for behavioral studies.

Traditionally, studies of cortical spatial representations have relied on the unit recording technique as a mapping tool (Merzenich et al., 1978: Kaas et al., 1979). This heroic mapping technique focuses on many individual cells, seeking to identify their receptive field properties. Optical imaging of intrinsic sig-

Received Dec. 8, 2000; revised May 4, 2001; accepted May 18, 2001.

This work was supported by the Grodetsky Center, by a grant from Bundesministerium für Bildung, Wissenschaft, Forschung und Technologie, by the Israeli Science Foundation, and by a grant from the Horace Goldsmith Foundation. We thank Dr. Jon Kaas for constructive comments on a previous version of this manuscript, Daniel Glaser for help in the experiments, Dov Ettner, Steve Leytus, and Chaipi Wijnbergen for excellent technical support, and Lenore Shoham for help with this manuscript. Special thanks to the neurosurgeon Dr. Zvi H. Rappaport, who participated in the human studies.

Correspondence should be addressed to Dr. Amiram Grinvald at the above address. E-mail: amiram.grinvald@weizmann.ac.il.

Copyright (C) 2001 Society for Neuroscience $\quad 0270-6474 / 01 / 216820-16 \$ 15.00 / 0$ nals (Grinvald et al., 1986; Frostig et al., 1990; for review, see Grinvald et al., 1999b), on the other hand, can be used to study spatial representation from a different perspective, presenting specific stimuli and seeking to identify the cortical columns that respond to them. Therefore, maps obtained by optical imaging avoid the sampling problem and make it easier to search for a needle in a haystack.

Several other reasons also motivated us to implement the intrinsic optical imaging method to monkey primary somatosensory cortex. First, cortical plasticity has been extensively studied in this brain region; a lot of data has accumulated regarding the reorganization of somatotopic maps in response to injury or experience (for review, see Kaas, 1991; Buonomano and Merzenich, 1998). Plasticity studies can benefit from the use of optical imaging because it can provide complete maps of large cortical areas in a short time repeatedly, reproducibly, and over a long period of time as demonstrated previously in the rat (Masino and Frostig, 1996; Polley et al., 1999), cat (Kim and Bonhoeffer, 1994), ferret (Chapman et al., 1996), and behaving monkey (Shtoyerman et al., 1995, 2000; Vnek et al., 1999).

Having accomplished high-resolution functional maps in the somatosensory cortex of the macaque, we were motivated to assess how difficult it is to obtain similar high-resolution functional maps of the human brain during neurosurgery. The pioneering efforts by MacVicar et al. (1990), Haglund et al. (1992), Toga et al. (1995), and Cannestra et al. (1998) were aimed at the challenging task of delineation of functional borders before tumor or epileptic focus or arteriovenous malformation (AVM) resections to minimize the potential damage that can be accomplished after the functional borders are properly delineated. To date, optical imaging as well as other traditional techniques (Pen- 
field and Boldrey, 1937; Kido et al., 1980) has been limited and therefore is not in routine use during neurosurgery. Therefore, there is a pressing need to improve fast intraoperative delineation of functional borders. Furthermore, gaining additional insight into the behavior of human microcirculation should assist the interpretation of functional maps obtained by functional magnetic resonance imaging (f-MRI); this noninvasive neuroimaging relies on signals from the cortical microcirculation similar to those of the optical imaging mapping performed here.

\section{MATERIALS AND METHODS}

The methods for preparing and maintaining the animals and the imaging setup and procedures were primarily similar to those described previously (Ts'o et al., 1990; Bonhoeffer and Grinvald, 1993; Grinvald et al., 1999b). These methods are briefly described below together with a detailed description of experimental and data analysis methods specifically developed for this study. We begin with the monkey experiments and proceed with the methodology used during the human studies.

\section{Experimental procedures}

Animals. Optical data for this study were obtained from five adult macaque monkeys (Macaca fascicularis) weighing 7-8 kg. In the first two monkeys we applied only median nerve stimulation, and in the other three we also used the mechanical stimulation paradigm as described below.

All surgical procedures were performed according to the National Institutes of Health guidelines. Anesthesia was induced with an intramuscular injection of ketamine hydrochloride $(10-15 \mathrm{mg} / \mathrm{kg})$, supplemented in some experiments with xylazine hydrochloride $(0.5 \mathrm{mg} / \mathrm{kg})$. Atropine sulfate $(0.05 \mathrm{mg} / \mathrm{kg}$, i.m.) was also given at that time to decrease salivation. After tracheal cannulation, the animal was placed in a conventional stereotaxic apparatus and was artificially respirated. Local anesthetic (lidocaine hydrochloride) was applied to wound and pressure points. Sustained anesthesia was achieved by a continuous intravenous infusion of sodium pentothal $\left(4 \mathrm{mg} \cdot \mathrm{kg}^{-1} \cdot \mathrm{hr}^{-1}\right)$. The animal was paralyzed with vecuronium bromide (Norcuron, $0.2 \mathrm{mg} \cdot \mathrm{kg}^{-1} \cdot \mathrm{hr}^{-1}$, i.v.). The drugs were supplied in dextrose-saline solution (sodium chloride, $0.9 \%$; dextrose, $5 \%$ ). The electrocardiogram and the electroencephalogram were continuously monitored. The end-tidal $\mathrm{P}_{\mathrm{CO}_{2}}$ pressure was maintained at $4-5 \%$ by adjustment of the tidal rate and volume. The rectal temperature was kept at $38^{\circ} \mathrm{C}$ using a heating blanket.

Optical imaging. A hole (diameter of $\sim 23 \mathrm{~mm}$ ) was drilled in the skull of the monkey, centered approximately over the medial part of the central sulcus, and a stainless steel optical chamber (internal diameter of $30 \mathrm{~mm}$ ) was cemented over the hole and sealed with wax. The dura was removed, and the chamber was filled with silicone oil and closed with a round glass cover plate. This arrangement minimized the movement of the cortex because of heart pulsation and respiration that otherwise severely hampers optical imaging.

The surface of the cortex was illuminated by two adjustable light guides that were attached to a Zeiss tungsten-halogen lamp $(100 \mathrm{~W})$. The light was passed through interference bandpass filters of different wavelengths. High-contrast images of the cortical surface and its vasculature were obtained using green light (filter with transmission at $540 \pm$ $15 \mathrm{~nm})$. For functional maps we used red light $(605 \pm 5 \mathrm{~nm})$ and focused the camera $300-600 \mu \mathrm{m}$ below the cortical surface.

One of two imaging systems was used to control the experiments and to record the images of the cortex. The first system, introduced by Ts'o et al. (1990), uses a slow-scan CCD camera (Photometrics, Ltd., Tucson, AZ) with a spatial resolution of $192 \times 144$ pixels and 12 bits of digitization. The second system (IMAGER-2001; Optical Imaging, Germantown, NY) includes a standard, high-quality video camera with resolution of up to $765 \times 574$ pixels. The camera is coupled to a differential video amplifier that obtains effective 12-bit digitization using 8-bit hardware and also provides an enhanced video signal in real time. The "enhanced video image" that is seen in real time with this system has been of great value during the clinical studies because it allowed us to get immediate feedback regarding the nature and the level of noise in the recording. Thus in some cases we were able to reduce the noise before the actual imaging session started.

The images were formed using the "tandem-lens" arrangement (macroscope) that was developed in our laboratory (Ratzlaff and Grinvald, 1991). This device is in essence a microscope (with low magnification) built out of two photographic lenses, coupled front-to-front, that together provide an unusually high numerical aperture. This system consequently has a very low depth of field (nominal $50 \mu \mathrm{m}$ ). It allowed us virtually to eliminate most artifacts caused by blood vessels on the surface of the cortex by smearing them over a large area (Malonek et al., 1990; Ratzlaff and Grinvald, 1991). The ratio of the f-numbers of the two lenses determines the magnification of the macroscope. We usually started imaging with a $50 \mathrm{~mm}$ lens on top and a $135 \mathrm{~mm}$ lens at the bottom $(2.7 \times$ minification). This lens combination provided a $22 \times 16 \mathrm{~mm}$ image that included almost all of the exposed area. We first ran a few trials with this magnification to localize quickly the somatosensory hand area. We then focused on this area and switched to a higher magnification (either $8 \times$ $6 \mathrm{~mm}$ field using two $50 \mathrm{~mm}$ lenses or a $16 \times 12 \mathrm{~mm}$ field using a $25 \mathrm{~mm}$ lens on top of a $50 \mathrm{~mm}$ one). To facilitate the on-line analysis of the results and to minimize data size, we usually defined an even smaller region of interest and discarded the rest of the image.

Electrical stimuli. To stimulate the median nerve, we attached a pair of electrocardiogram (EKG) electrodes (Promedico, Tel-Aviv, Israel) to the cleaned skin above the median nerve at the wrist. These electrodes were connected to an isolated current source through which we passed short $(0.5-1 \mathrm{msec})$ current pulses. The nerve was localized by searching across the wrist for the point of minimal threshold for thumb twitch response. This was done before paralyzing the animal. The current was set to a level of just above the threshold for thumb twitch. For optical imaging, we typically applied trains of 20 pulses at $10 \mathrm{~Hz}$.

Mechanical stimuli. Weak mechanical tactile stimulation of the fingers was achieved by applying trains of low air pressure pulses to small flexible transducers (Biomagnetic Technologies, San Diego, CA) that displace the skin surface at the location of contact. One stimulator was attached to each finger on the glabrous side of the distal phalanx. This type of tactile stimulus appears rather mild on our own fingers, has been used in magnetoencephalograph studies on humans (C. Gallen, personal communication), and was not further quantified. In some of the experiments we also attached stimulators to other phalanges and to the pads of the palm or to the wrist. The transducers were connected to a regulated air pressure source via a set of fast solenoid valves (General Valve, Fairfield, NJ) using Teflon tubing. Voltage pulse trains were generated by a computer-programmable multichannel pulse generator (Master-8; AMPI, Jerusalem, Israel) and were delivered to the solenoid valves via an electronic relay to produce the air pressure pulse trains. A typical stimulus was a single train of $10 \mathrm{msec}$ pulses given for $2 \mathrm{sec}$ at $10 \mathrm{~Hz}$ (total of 20 pulses).

Data acquisition. The stimulus duration was $2 \mathrm{sec}$ in most recordings, and the interstimulus interval was $7-15 \mathrm{sec}$. The acquisition of cortical images started typically $\sim 0.5 \mathrm{sec}$ before stimulus onset and lasted for $4-7$ sec. During this period we collected 5-16 frames. When the slow-scan CCD camera was used, these were individual frames (accumulating light for $\sim 500 \mathrm{msec}$ each time), and when the video camera was used, each of these frames was the result of an on-line summation of 10-15 video frames. To reduce the noise associated with respiration and heart beat, the stimulus onset and the data acquisition were synchronized to the heart pulsation and to the respiration cycle.

The complete stimulus set was presented 10-128 times, each time in a different randomized sequence, to average out stimulus-dependent aftereffects. The response to each stimulus was summed over trials on-line and saved on the disk of the computer in blocks of 5-16 repetitions.

Electrical recording. To confirm the optical results, the optical imaging session was followed, in some experiments, by targeted recordings of single-unit and multiunit activity. We used an optical chamber specially designed for targeted electrical recordings into optically imaged functional domains. This new design enables fine, three-dimensional manipulation of an electrode within the optical chamber. Details of this apparatus will be described elsewhere (Grinvald et al., 1999a) (A. Arieli and A. Grinvald, unpublished observations). We used low-impedance $(\sim 0.6 \mathrm{M} \Omega$ at $1 \mathrm{kHz})$ glass-coated tungsten microelectrodes with tip diameter of $\sim 15 \mu \mathrm{m}$. We tried to make the penetration angles as close to perpendicular to the cortical surface as possible. Single units were detected and isolated using a window discriminator. All of the spikes with an amplitude higher than approximately three times the noise level were recorded as multiunit activity. The recordings were made in the superficial layers $(\sim 300-800 \mu \mathrm{m})$ at three different depths for each penetration. The stimuli and the stimulus application protocol were the same as for the optical recording. In each recording the stimulus set was repeated eight times, with an interstimulus interval of $10 \mathrm{sec}$. 


\section{Data analysis}

Computing the functional maps. As described above, the optical data from each block (sum of 5-16 trials) were kept as temporal sequences of 5-16 frames for each stimulus condition. In the first step of data analysis, we summed for each stimulus the frames from all of the blocks and all (or a range) of the temporal frame sequence. This summation resulted in a single cortical image per stimulus condition.

The functional maps were produced from these images by dividing the image from one stimulus condition (or an average of two or more conditions) by the image for another stimulus condition (or average of a few conditions). This division removes the effect of uneven illumination and produces a map that depends only on the difference in cortical reflectance between the two conditions (or sets of conditions). In all of the imaging sessions, we included a "blank" (no-stimulus) condition. Most of the maps in this study were produced by dividing the image from a particular stimulated condition by the image from the blank condition ("single-condition maps"). In addition we also computed maps by dividing images from different stimuli ("differential maps").

We also examined the development of the optical signals in time. This was done by dividing each of the frames from the temporal frame sequence of a given stimulus condition by the corresponding frame from another stimulus condition (usually the blank).

When attaching the stimulators to the different skin locations, we attempted to make all stimuli as identical as possible. However, there is still a possibility that some stimuli were more effective than others. The imaging results showed that the amplitudes of the optical signals evoked by equivalent stimuli to different fingers were quite similar in most cases. However, in some cases there were significant differences in these amplitudes. Thus, when the maps for different stimulus locations were compared or combined, we first normalized each of the maps. Normalization was done by subtracting from each map its median value and dividing the result by the amplitude of maximal activation found in the maps. The maximal activation value was computed from a smoothed version of the map (low-pass filtering with a Gaussian filter, $\sigma=120 \mu$ ) so that high-frequency noise did not affect the obtained value.

Composite finger maps. We combined the information obtained by stimulating each finger separately into a single somatotopic map using two different methods. The first method uses contour lines surrounding a given signal level. The single-finger maps were smoothed with a Gaussian low-pass filter $(\sigma=120 \mu)$ and normalized as explained above, and contour lines at the level of $30 \%$ of the peak activation level were computed for each finger. The contours were color-coded and superimposed over the vascular cortical image.

The second method of creating a composite map uses a "winner-takesall" rule. The winner-takes-all map (WTA map) was computed from the smoothed and normalized single-finger maps in the following way. First we assigned a color for each of the fingers (see the drawing of the hand at the bottom of Fig. 4). For each pixel in the image we compared the response to all of the fingers and assigned a color according to the finger that gave the strongest response. The intensity value was determined by the response amplitude. To combine this map with the raw image of the cortex, we applied a threshold such that the color of each pixel with activity below the threshold was replaced with the gray level of the corresponding green-illuminated cortical image (see Fig. 6, bottom).

Noise reduction using "first-frame subtraction." The slow noise interference seems to be more dominant in the current study in comparison with our previous experience in the visual cortex. This difference could reflect a genuine difference between the hemodynamic characteristics of the somatosensory and the visual cortices. This possibility gets some support from reports of high-amplitude slow fluctuations in the somatosensory cortex of the rat (Chen-Bee et al., 1996). A similar problem was reported also in the auditory cortex of the rat and the guinea pig (Bakin et al., 1996). In many recording sessions reported here, traditional analysis of the data, without the first-frame subtraction, barely revealed the functional maps (Fig. 1A). Yet slow noise can be nearly eliminated by proper analysis. The first-frame procedure used here has been rarely used, in spite of the fact that it can "rescue" otherwise useless data. Therefore, we elaborate on this topic here. Various noise sources with different spatial and temporal characteristics interfere with the optical intrinsic signals. The quality of the maps is often limited by slow fluctuations that are presumably associated with slow changes in cortical hemodynamics as described in Results. These fluctuations are sometimes a-periodic and sometimes nearly periodic with a frequency of $\sim 0.1 \mathrm{~Hz}$ typical of the "vasomotion" phenomenon (Schiff, 1854; Mayhew et al., 1996). In some of the imaging sessions reported here, such slow noise severely impaired
A
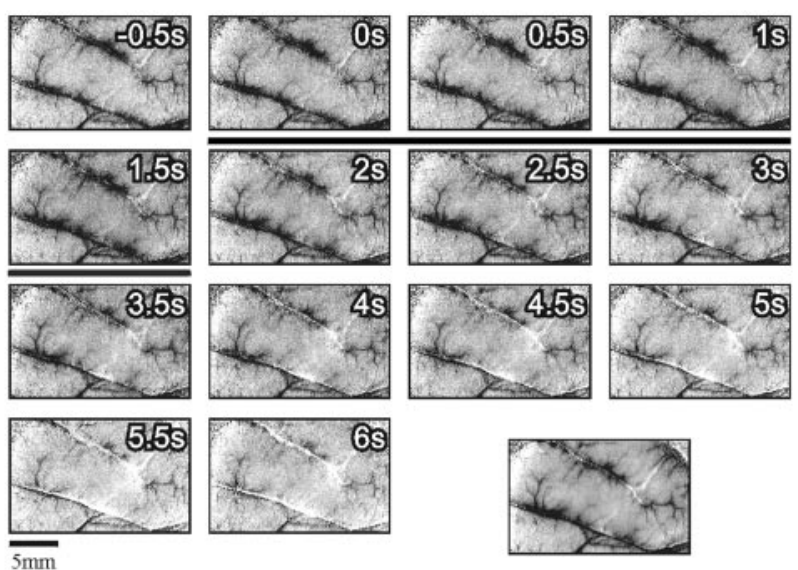

B
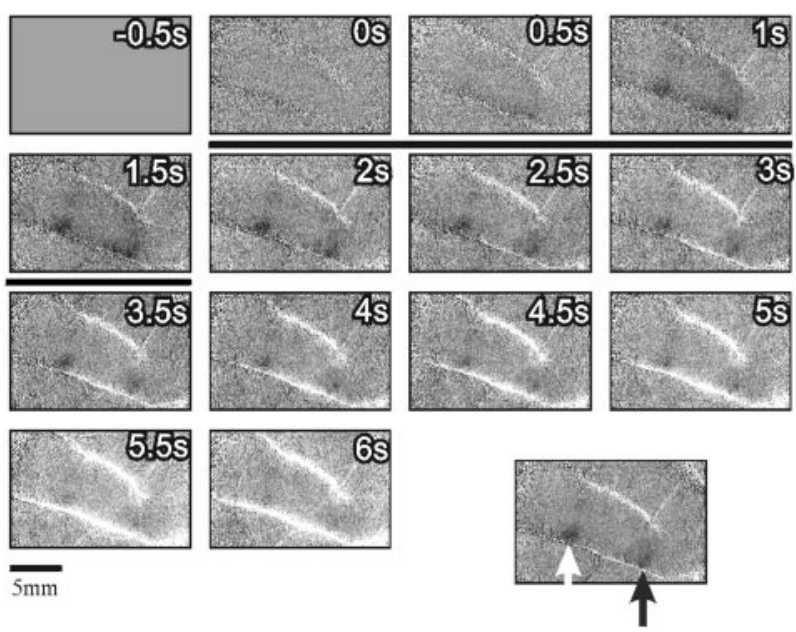

Figure 1. Improvement of functional maps using first-frame subtraction to remove common slow vascular noise. $A$, Image sequence obtained with mechanical stimulation of a single fingertip (finger 1) in monkey M3. Each frame was divided by the corresponding frame from the blank condition and scaled such that the full gray scale corresponds to a fractional change of $1.7 \times 10^{-3}$. The specific finger activation is not seen here. Instead, an almost constant noise pattern is evident. This pattern is not related to the stimulus because it appears at the time of the first frame, preceding the stimulus onset (stimulus duration is marked by a black horizontal bar). The image at the bottom right is the average of frames 4-9 (1-4 sec after the stimulus) of the above sequence. Because the dominant noise pattern exists in all of these frames, this averaging does not eliminate it. $B$, The same image sequence from $A$ after subtracting the average of the first three frames from all subsequent frames. Here the stimulus-evoked activation is evident. Again, the image at the bottom right is the average of frames 4-9 of the above sequence. The dark patch on the right (black arrow) is the finger 1 domain. The dark patch on the left (white arrow) was activated also with stimulation of other fingers. Further discussion of these activation patterns is given below. The full gray scale in all images corresponds to a fractional change of $1.7 \times 10^{-3}$. $s$, Seconds.

the visibility of the functional domains. To improve the maps and remove most of the common slow noise, we introduced the procedure of firstframe subtraction. This procedure uses data obtained just before the stimulus onset to correct the subsequent images. Because of the low temporal frequency of this noise, the resulting patterns do not change much over the acquisition of a single response. Because the noise does change from trial to trial, dividing one condition by another (or by the blank) will not correct for it. When the amplitude of such noise is relatively high, it might dominate the maps; a time sequence of differential or blank-corrected images that should show the development of the maps in time will appear almost constant, because the evolving func- 
tional map is masked by this slow vascular noise (Fig. $1 A$ ). In such cases, it is beneficial to start data acquisition some time before stimulus onset and to collect one or more prestimulus frames. The resulting image will contain the (almost) constant noise pattern and not the evoked signal. Subtracting this image (or average of images) from each of the subsequent images will then remove most of the common slow noise and will help reveal the functional map.

Figure $1 B$ illustrates the remarkable improvement of functional maps that this approach can yield. This is the same sequence shown in Figure $1 A$, after subtracting the first frame. Here the evoked signal is evident, and the somatotopic activation is revealed. The case shown here is an extreme (but not atypical) example of the common slow-noise effect. Some of the recordings were much less affected by this type of noise, but for uniformity of the display, we used first-frame subtraction in all images shown below.

Despite the impressive improvements, which can often be achieved with first-frame subtraction, this procedure also has its disadvantages, the most important of which is the introduction of high-frequency noise into the maps. This results from the fact that a single (noisy) image is subtracted from all other frames, and therefore the noise of the first frame is introduced into the analysis in a way that subsequent averaging over frames will not cancel out. Therefore, we used up to four nostimulus frames to reduce this high-frequency noise.

As noted above, the relative effect of the slow noise varies across experiments and even during a single experiment. This variability is possibly related to factors like the physiological state of the animal, the anesthesia level, etc. We have also discovered that quite often the body posture has a very strong effect on this signal. Using the enhanced video system, we could visualize these signals in real time. They often appeared as large (few millimeters) "clouds" that slowly scan the cortex or move in circles. Sometimes we could greatly improve the situation by simply elevating or lowering the body by a few centimeters (relative to the head) or by other changes to the body posture.

Analysis of electrical recordings. The spike trains from each recording (eight trials) were used to generate peristimulus time histograms (PSTHs) with $10 \mathrm{msec}$ bins. The PSTHs were displayed along with the raw data ("raster display"). In addition we computed the tuning curves for each recording by counting the spikes (summing over trials) in the 2 sec stimulation period. The baseline firing rate was computed by averaging the spike counts from the $0.5 \mathrm{sec}$ before stimulus onset over all stimuli. The tuning curves from the different penetrations were normalized to have a common maximal value.

Human subjects. The 15 subjects who participated in this study were adult patients who had to undergo brain surgery for tumor or AVM resection. They all signed informed consent forms according to regulations of the internal review board of the hospital.

Intraoperative optical imaging. All of the experimental procedures have been conducted in conformance with the policies and principles contained in the federal policy for the protection of human subjects, in the declaration of Helsinki, and the protocol approved by the hospital internal review board. Both surgery and imaging were done under general anesthesia using isofluorane. A transparent perspex window was placed on the exposed cortical surface to stabilize it. The cortex was illuminated by two adjustable light guides attached to a Zeiss tungsten-halogen lamp $(100 \mathrm{~W})$. Cortical images were obtained using a single macrozoom lens attached to a digital imaging system. Typically we imaged an area of $\sim 50 \times 50 \mathrm{~mm}$. To obtain high-contrast images of the cortical surface and its vasculature, we used green light $(540 \pm 15 \mathrm{~nm})$ and focused the camera on the surface. To image the functional maps, we used red light $(605 \pm 5$ or $630 \pm 5 \mathrm{~nm})$ and focused the camera $300-600 \mu \mathrm{m}$ below the cortical surface.

The camera was mounted on a fine $x-y-z$ translator that was attached to a heavy Zeiss surgical binocular stand, replacing the binocular. It was essential to reduce camera vibrations relative to the patient cortical surface. Therefore we built a special stabilizing construction around the stand and its flexible arms. With this construction the camera could be first manipulated coarsely using the degrees of freedom of the arm of the Zeiss stand, and then its position was locked to prevent further movements or vibrations. Additional fine positioning could be performed after locking the system using the $x-y-z$ translator and the $z$ translator attached to the binocular stand. To reduce vibrational noise further, the camera itself was rigidly attached and tightly locked to the patient head holder, after focusing on the target area. The electrical and mechanical stimuli used were very similar to those we optimized in the macaque studies as described above.
Data acquisition. The stimulus duration was $2 \mathrm{sec}$ in most recordings, and the interstimulus interval was $7-15 \mathrm{sec}$. The acquisition of cortical images typically started $\sim 0.5 \mathrm{sec}$ before stimulus onset and lasted for 4-7 sec. During this period we collected 5-16 frames. Each of these frames was the result of an on-line summation of $10-15$ video frames. To reduce the noise associated with respiration, in some patients stimulus onset and data acquisition were synchronized to the patient respiration cycle. The complete stimulus set was presented 5-16 times, each time in a different randomized sequence, to average out stimulus-dependent aftereffects. To optimize the off-line image processing, each of the individual responses was saved separately on the disk of the computer.

Electrical recording of human surface-evoked potentials. To confirm the functional map obtained by optical imaging, we followed the imaging session in some patients with electrocorticographic recordings. We used surface electrode arrays (Ad-Tech; up to $4 \times 5$ electrodes; $1 \mathrm{~cm}$ interelectrode spacing) to record cortical surface-evoked potentials (SEP) from multiple sites. In addition to the electrode array, we inserted a strip of four electrodes under the dura and the skull lateral to the exposed cortex. These electrodes (electrically connected to each other) served as a common reference to all of the recordings shown in one figure (see Fig. 15). We used the same stimuli (both electrical and tactile) that was used for the imaging, applying single pulses at a rate of $2-5 \mathrm{~Hz}$. The signals were amplified and filtered $(10-1000 \mathrm{~Hz})$ using Grass amplifiers and recorded on a personal computer after 12-bit digitization $(1700 \mathrm{~Hz})$. The responses to 128-256 repetitions of each stimulus were averaged on-line and saved to disk. At least two such sets of data were collected for each recording.

\section{RESULTS}

The area explored in this study was the surface of the left postcentral gyrus of the macaque monkey focusing on the somatosensory hand representation. In the macaque monkey, the exposed part of the gyrus contains most of Brodmann's areas 1 and 2. This region is outlined on the schematic macaque brain in Figure $2 A$, which also shows the vascular pattern imaged in one of the experiments. All subsequent images are at the same orientation or slightly rotated so that the central sulcus parallels the bottom of the image.

\section{Single-finger time course}

To study the detailed somatotopy of the hand area, we used the pneumatic tactile stimulation system (see Materials and Methods) in three of the monkeys. The series of frames in Figure $2 B$ shows the time course of the intrinsic signals evoked by stimulation of a single finger (finger 3). Two major components are evident in the response. The first component is darkening in a finger-specific stripe of cortex traversing the gyrus (the finger domain, black arrow in Fig. $2 B, 2 s$ ) and in a second, nonspecific patch [white arrow, same as in Fig. $2 B$, bottom right for finger 1 activation; further details about this domain are below (see Fig. 7)]. This darkening peaks at the fifth frame (Fig. 2B, 1.5-2 sec after stimulus onset) and decays slowly. In addition there is slow brightening of the whole imaged area, which is strongest in the large blood vessels (see Fig. $2 B$, bottom right, the vascular pattern). This brightening rises steadily until the end of the recording. This time course appears approximately similar to that observed in our previous studies of macaque V1. Stimulation of the other fingers resulted in a similar activation pattern, with a different specific strip for each of the fingers, and a similar global brightening (Fig. $1 B$ showing the response to finger 1 stimulation in the same recording session). It is well established that such darkening corresponds to electrically active areas, whereas the diffused brightening in the parenchyma and in the large vessels corresponds to enhanced blood flow during the hyperoxygenation phase, which spreads well beyond the electrically active area (Frostig et al., 1990; Malonek and Grinvald, 1996; Grinvald et al., 2000). 


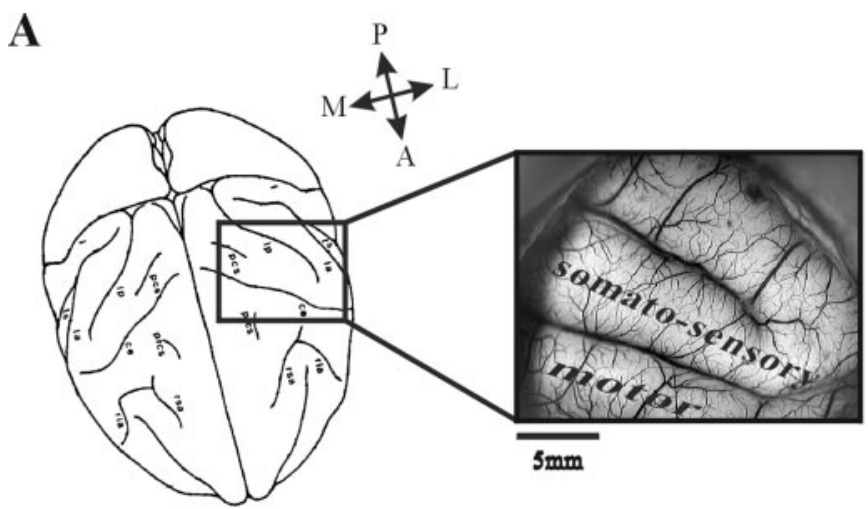

B

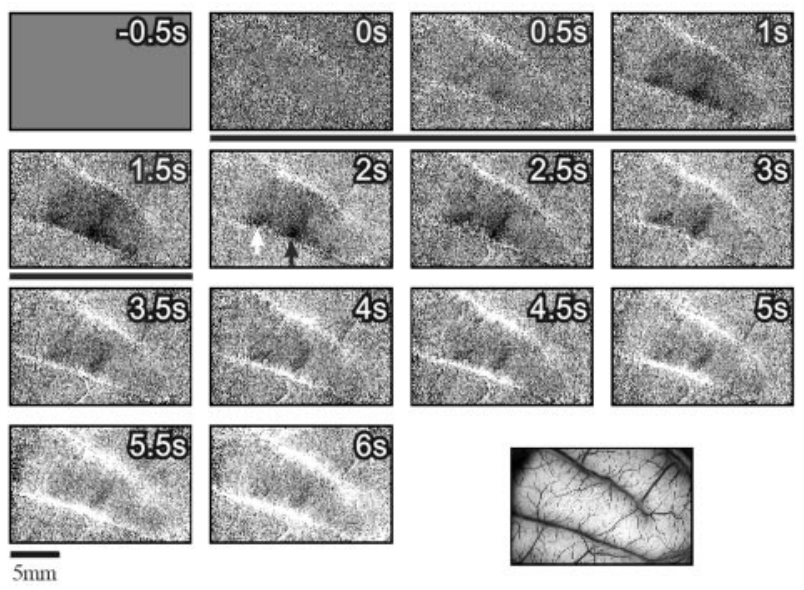

Figure 2. Time course of optical response to mechanical stimulation of a single finger. $A$, Left, A schematic dorsal view of the macaque brain outlining the cortical territory explored in a typical somatosensory imaging session. Right, Image of the cortical surface (left hemisphere, monkey M3) illuminated with green light $(540 \mathrm{~nm})$ to emphasize the blood vessel pattern. The somatosensory and motor areas are marked. The anterior part of the somatosensory strip is Brodmann's area 1, and the posterior part is area 2. $B$, An image series of the left hemisphere in monkey M3 showing the temporal development of the optical response to a weak mechanical indentation stimulation of finger 3 of the right hand. Each frame represents $500 \mathrm{msec}$ of summation of collected video frames. The image at the bottom right shows the corresponding vascular image taken with green light (for general orientation, see $A$ ). The finger was stimulated at the distal phalanx using the pneumatic stimulator described in the text (Materials and Methods). Air pressure pulses $(10 \mathrm{msec})$ were delivered for $2 \mathrm{sec}$ at a rate of $10 \mathrm{~Hz}$. The stimulus started concurrently with the acquisition of the second frame (stimulus duration is marked by a black horizontal bar). To obtain these images, we divided each image from the sequence obtained during stimulus application by the corresponding image from the blank (no-stimulus) condition. This procedure, in addition to normalizing for the illumination pattern, nearly eliminates the signals coming from respiration and heart pulsation because data acquisition was always synchronized to both of these cycles. First-frame subtraction was applied. Here and in the following figures, activity shows up as darkening in the image. The full gray scale corresponds to a fractional change of $1 \times$ $10^{-3}$. The images from 45 repetitions of the stimulus (and blank) presentations were averaged. $A$, Anterior; $L$, lateral; $M$, medial; $P$, posterior.

\section{Single-finger maps}

To emphasize the specific finger activation, we summed frames 4-9 (1-4 sec after the stimulus) from sequences like the one shown in Figure 2B. The results are shown in Figure 3. Here and in the following maps, we focused on the activated part of the postcentral gyrus. [Similar maps from two other monkeys are

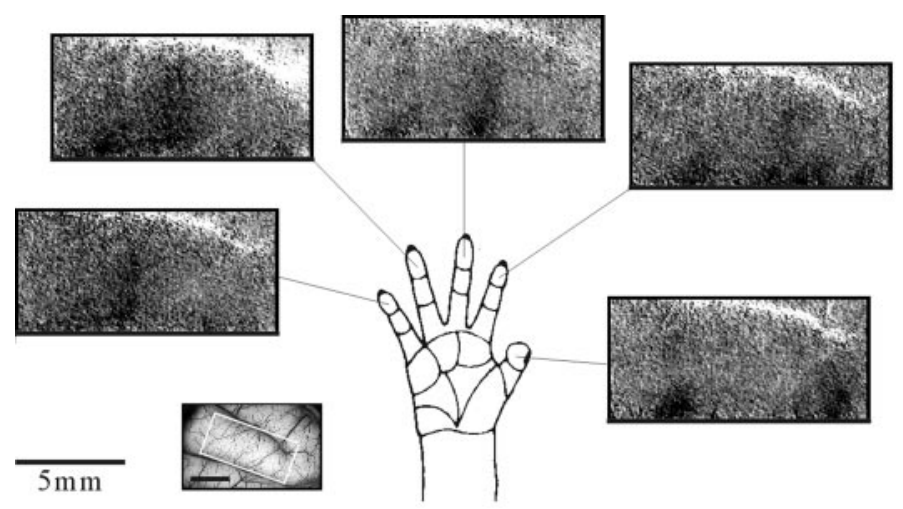

Figure 3. Cortical representation of single fingers. Image sequences like the one in Figure $2 B$ were obtained for stimulation of each of the five fingertips. Frames $4-9$ of each series (1-4 sec after the stimulus) were averaged to produce the single-finger activity maps shown here. The resulting images were rotated and cropped to focus on the activated part of the postcentral gyrus. The white rectangle on the vascular imaging at the bottom outlines the cropped area. The maps in all subsequent figures are shown at this orientation. The five images surrounding the hand drawing show the areas activated by mechanical stimulation of the five fingers. A somatotopic organization of the hand representation is evident, with the thumb on the right (anterolateral) and the little finger on the left (posteromedial). In addition to the finger-specific activations, there is a patch of cortex just medial to the hand representation that is activated by all stimuli, including a weak activation by finger 5 (see also Figs. 4, 6, in another monkey). Further discussion of this domain is given below (see Fig. 8). The full gray scale corresponds to a fractional change of $1 \times 10^{-3}$.

shown below (see Figs. 4, 12).] These maps show that the optical signals evoked by the tactile stimulation of a single finger were strongest in a narrow transverse strip $(\sim 1 \times 4 \mathrm{~mm})$ across the postcentral gyrus. As expected, we found a sequential organization of the finger representations, with the thumb most anterolateral and the little finger most posteromedial along the gyrus. In addition to these finger-specific domains, the maps show that another patch of cortex just medial to the hand representation was activated by all stimuli. This issue is discussed below (see Fig. 8 for additional details).

Although the centers of the different finger domains formed a clear somatotopic succession along the gyrus, there was considerable spatial overlap between the optical signals evoked by stimulation of neighboring fingers. The largest overlap was between fingers 3,4 , and 5 , and the smallest was between fingers 1 and 2 . The response to finger 1 stimulation was consistently almost isolated, with very little spatial overlap with the response to finger 2 stimulation.

The postcentral gyrus in the macaque monkey contains most of Brodmann's areas 1 and 2. Area 1 occupies the anterior part of the gyrus (approximately one-half to two-thirds of it), and area 2 occupies the remaining posterior part. In our experiments we did not use independent measures to determine the exact location of the border between areas 1 and 2. Still, some features of the optical maps fit nicely with the presumed areal border. In most cases the single-finger domains were not uniform across the gyrus. The activation was generally strongest in the anterior part (bottom of the images) and weaker in the posterior part. In some cases there was even a gap between the anterior and the posterior activations (e.g., see Fig. 6), and in some cases there was a difference in the mediolateral position of the activation by the same finger in the two parts of the gyrus. 

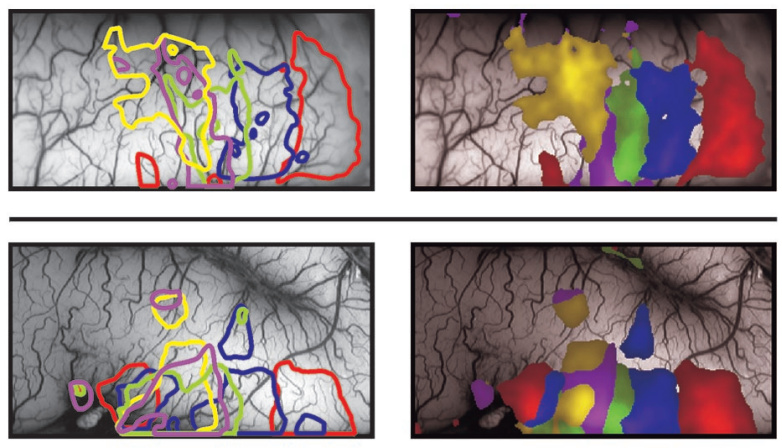

M5
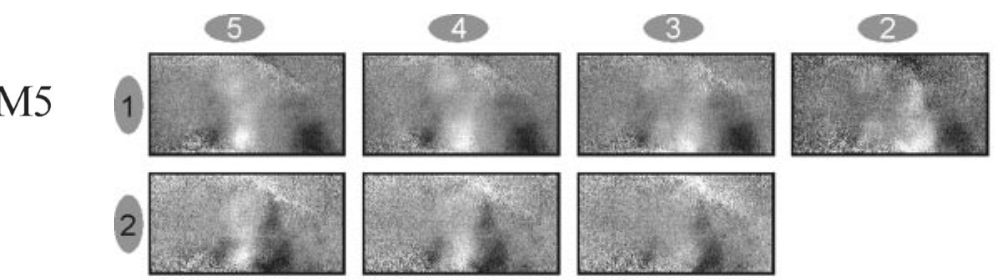

M4
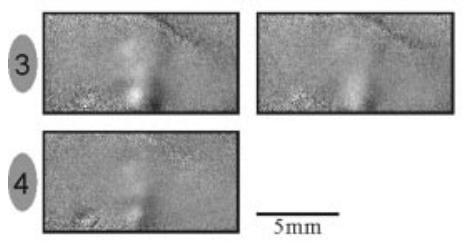

$5 \mathrm{~mm}$

Figure 5. Differential finger maps. The five single-finger maps from monkey M4 were used to generate the differential maps for each pair of fingers. The top row shows the images resulting from subtracting the maps of fingers 2-5 from the finger 1 map; the second row shows the finger 2 map minus the maps of fingers $3-5$, and so on. Before the images were subtracted, each map was normalized as explained in Materials and Methods, so that the maximal activation (defined as the minimal value in the smoothed version of the map) was mapped to 1.0 and the median value was mapped to 0.0. Next, the resulting differential maps were all scaled such that the range ( -1 to 1 ) would span the full gray scale.

Figure 4. Somatotopic organization of the hand area in three monkeys. Left hemisphere finger maps for right-hand stimulation were obtained in a way similar to the way in which the ones in Figure 3 were obtained. The maps from all three monkeys are summarized here in two formats. Left, The images show contour maps from the three monkeys. Contour lines at the level of $30 \%$ of peak activation for each finger are superimposed on the surface vasculature image (imaged with green light). The thin contour lines were computed from a smoothed version of the maps (low-pass filtered with a Gaussian filter, $\sigma=120 \mu$ ). The contour for each finger is colored according to the color code shown in the hand drawing below the maps. To facilitate a comparison with the second type of analysis shown on the right, the WTA patches are also superimposed. Right, The images show WTA maps calculated from the same data. The information from the individual finger maps is integrated here using a winner-takes-all rule. The color of each pixel is determined by the finger that gave the strongest response, using the same color codes used for the contours. The intensity encodes the amplitude of the response to the "winning" finger. Only pixels in which this response was $>30 \%$ of the peak activation were colored. The other pixels show the underlying vascular pattern. Also see Figure 6.

\section{Composite finger maps}

Some aspects of the overall organization of the mapped area are easier to study when the information is condensed into a single map. We used two different methods to combine the information from the five single-finger maps into a single image, as explained in Materials and Methods (here we assumed that the response at the peak of the activated area should be the same, and such a simplification may not always be justified). Figure 4 shows these composite maps from the three monkeys in which we mapped the responses to tactile finger stimulation. The left images show the contour-line maps, and the right images show the corresponding WTA maps. The two types of presentations emphasize complementary aspects of the data. The contour maps show the overlap between the evoked signals, and the WTA maps parcel the cortex according to which stimulus was most effective in each part. The somatotopic organization is clear in both types of presentations.

Because of the (arbitrary) threshold that we selected, the two sets of maps reflect mainly the organization of the anterior part of the gyrus (presumed area 1), where the signals had higher amplitude. The fainter patches of activity in the posterior part of the or the WTA maps at this threshold level. The relation between the contour lines and the original activation maps can be seen (see Fig. 6) when the individual contours are superimposed on the raw finger maps.

The WTA maps show that, in general, finger 1 had the widest territory and fingers 3-5 had the narrowest. It is important to note that this does not necessarily imply that the stimulation of finger 1 evoked activity over a larger cortical area. In fact, the contour maps (Fig. 4) and the raw finger maps (e.g., see Figs. 3, 6, 12) show that the size of the activated area was approximately the same for all stimuli. The domains of fingers 3-5 appear as narrow stripes in the WTA maps not because of a narrower activation area but because of the larger overlap between the activated areas. This is also demonstrated by the differential finger maps that are described below.

\section{Differential finger maps}

It is common practice in optical imaging studies to use differential maps in which the raw images from pairs of "orthogonal stimuli" are divided to show the difference in activation. Equivalently, blank-divided images can be subtracted (Bonhoeffer et al., 1995; Shoham, 1997). The maps discussed so far were all true singlecondition maps, i.e., normalized only by the blank (no-stimulus) condition. Figure 5 shows the differential maps from monkey M4. For each pair of fingers the normalized single-finger maps were subtracted to create the maps shown here. The substantial overlap between the activated areas of neighboring fingers that was discussed above on the basis of the single-condition maps is evident here. It is manifested in the small amplitude of differential maps from neighboring fingers (Fig. 5, images on the diagonal). The overlapping signals nearly cancel each other, and the resulting map is mainly faint gray. The only exception is the finger-1/ finger-2 map (top right image). When the differential maps are computed from non-neighboring fingers (above-diagonal maps), there is little interference, and the resulting maps span the full black-to-white gray scale. gyrus (presumed area 2) were not fully captured by the contours 
ODD BLOCKS
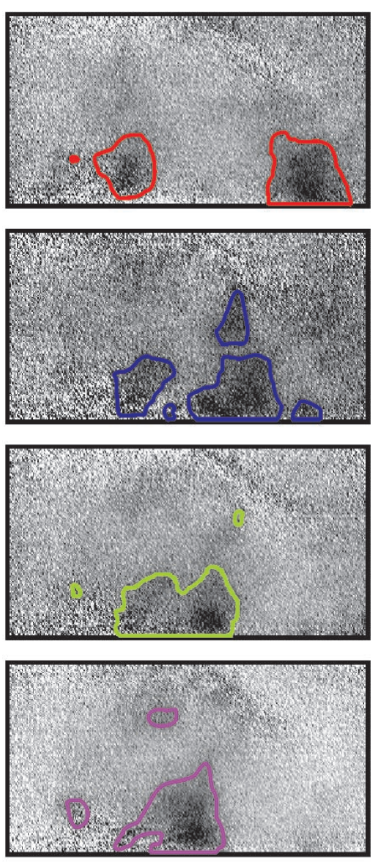

Figure 6. Reproducibility of the finger maps. The left and middle columns show two sets of single-finger maps (rows 1-5 from the top) and WTA maps (bottom row) from independent trials in the same cortex (monkey M4). The finger 1 map is at the top, and the finger 5 map is on row 5. These data were collected in 24 blocks of five trials each. For the reproducibility test, these images were divided into two sets of alternating blocks, and the functional maps were computed separately from each of these sets, containing 12 blocks. The average of the two left maps in each row creates the map in the right column. To aid in the comparison, the contour lines that were computed from the full data set were superimposed on all the maps. The color code is the same as that in Figure 4. The full gray scale corresponds to a fractional change of $1.2 \times 10^{-3}$.
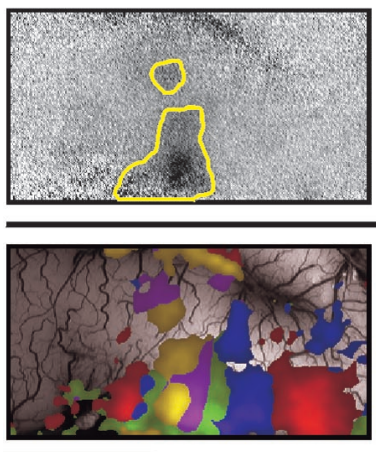

$5 \mathrm{~mm}$
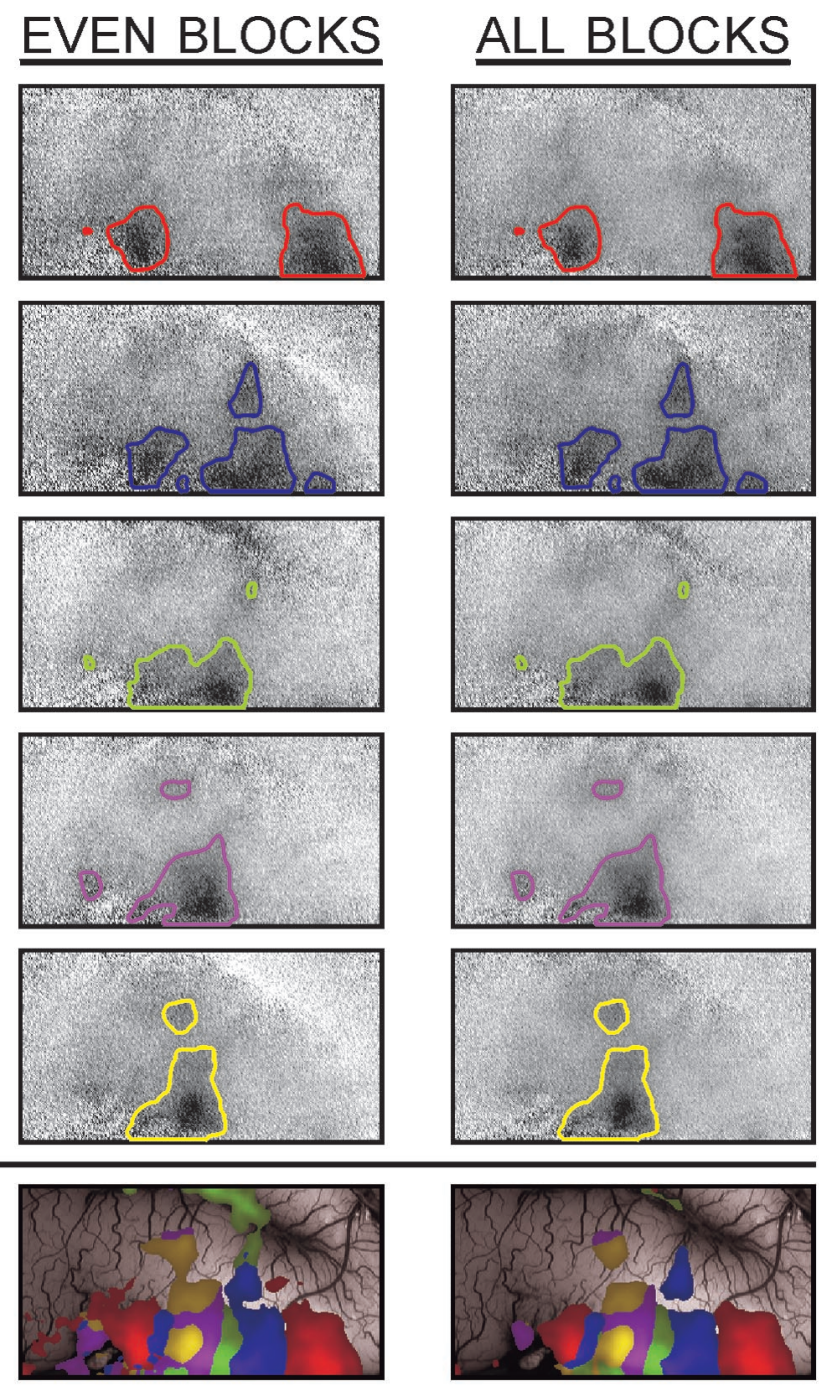

\section{Reproducibility of the maps}

To assess the reliability of the optical maps, we performed reproducibility tests. A typical example is shown in Figure 6 that shows the single-finger maps and the WTA maps from monkey M4. The maps in the right column are from a full recording session of 110 trials. The same data were split into two sets of alternating independent blocks, and the two sets of corresponding maps are shown in the left and middle columns. As expected, these maps are somewhat noisier than the average over all blocks, but the similarity between the two independent sets of maps is evident, confirming the reproducibility of the maps.

\section{Electrophysiological confirmation of the optical maps}

Targeted single-unit and multiunit recordings were used to confirm the optical results. At the end of the imaging session, we inserted microelectrodes into several identified loci in the imaged area. In each locus we recorded single-unit and multiunit responses to the same stimuli that were used for imaging (pneumatic stimulation of each of the finger tips). Multiunit data from such recordings are shown in Figure 7.

The raw data from one penetration are shown in Figure $7 A$. The electrode here was placed into the optically identified finger 1 area. The spike trains shown in the raster displays show the clear selective response to stimulation of finger 1 , as predicted from the optical maps. There was no response, or even slight inhibition, to stimulation of the other fingers. Furthermore, the response to the finger 1 stimulation showed a strong entrainment of the spikes to the stimulus pulses. The spikes were tightly locked to the stimulus, and almost all of the spikes during the response fell into single $10 \mathrm{msec}$ bins of the PSTH.

These multiunit data together with recordings from three other penetrations in the same monkey (M3) are summarized as tuning curves in Figure $7 B$. The second penetration was around the border of the finger 2 and finger 3 domains. The spike data, like the corresponding optical data, show a clear response to both fingers at approximately the same amplitude, with little response to the other fingers.

The third penetration is approximately at the center of the finger 5 domain. The cells recorded here responded best to stimulation of finger 5, but there was also a notable response to stimulation of the neighboring finger 4. This is also consistent with the overlapping activation seen in the optical maps.

The last recording was in the medial patch of cortex, which was activated by stimulation of all of the fingers. This patch and the results from this recording are discussed below. 
A

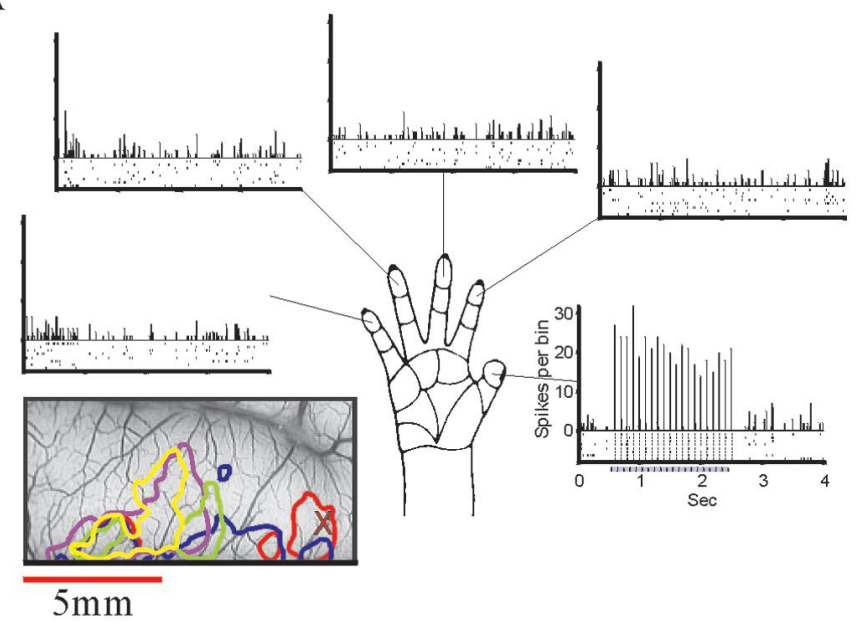

B

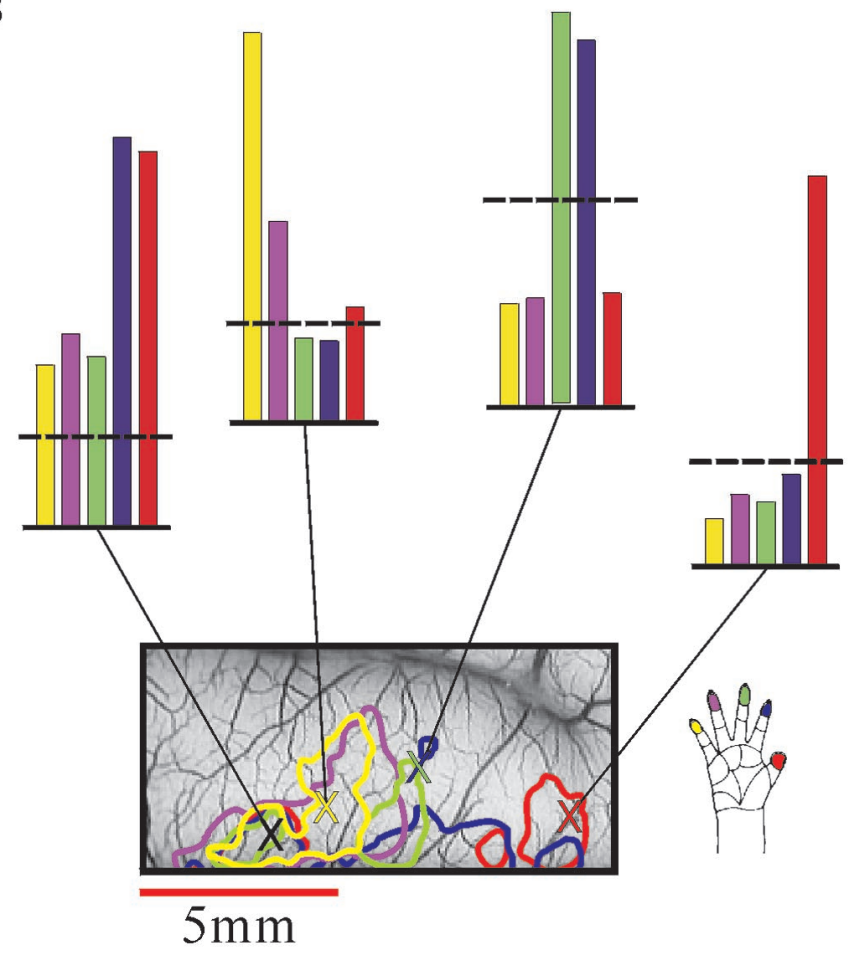

Figure 7. Targeted electrophysiological confirmation of the optical maps. $A$, The optical maps were used to guide electrode penetrations into optically identified loci. The data in this figure are from a recording in the finger 1 area (as determined by optical imaging) of monkey M3. [The penetration site is marked on the vascular image (bottom left $t$ by a red $X$.] The contours that summarize the imaging results (see Fig. 4) are also superimposed on the vascular image (below the left spike train). The stimuli used were the same as in the imaging session (at each of the 5 fingertips, $2 \mathrm{sec}$ at $10 \mathrm{~Hz}$ ). The data recorded from the site representing digit 1 show multiunit responses to stimulation of fingers 1-5 (right to left, counterclockwise). The spikes collected in eight trials for each stimulus are shown in the raster displays (bottom), and the resulting PSTHs (10 $\mathrm{msec}$ bins) are shown on the top. Stimulation of the thumb elicited a strong response with clear entrainment of the spikes to the stimulus pulses (right spike train). Stimulation of the other fingers elicited no response or even slight inhibition (e.g., digits 2-5 particularly during the response onset). The specificity of the response to finger 1 stimulation is very clear, confirming the optical results. $B$, The multiunit data from $A$ are summarized here together with data from the three other penetrations, one on the border of digits 2 and 3 (second histogram from the right), one for digit 5 , and one from the common patch. The contour finger map from Figure 4 is reproduced here, with marks ( $X$ symbols) at the four electrode
The single-unit data were very similar to the multiunit data (obviously with fewer spikes), including the multifinger responses.

\section{The "common patch"}

As described above, the tactile stimulation of single fingertips usually activated two separate patches of cortex along the postcentral gyrus. The more lateral part, the specific finger domain, was different for each finger. These patches make up the somatotopic map of the fingers that was described in detail above. The secondary activated patch was common to all of the fingers and was located posteromedially to the finger 5 representation. This common patch was observed in each of the three monkeys in which we mapped the responses to the rather weak tactile stimulation (e.g., see Figs. 3, 6, 12). The activation of this patch was strongest when finger 1 or 2 was stimulated and weaker for the other fingers. This is seen most clearly in the differential maps (Fig. 5); the common patch appears black (more active) in all of the maps in which the images from stimulation of fingers 3-5 were subtracted from the fingers 1 and 2 images (Fig. 5, top two rows, apart from the rightmost image on the top) and gray (equal activation) in the other maps. In one animal, activation was evoked almost exclusively by finger 1 stimulation (see Fig. 12). To illustrate the similarity of the architecture of this domain in the three studied animals, we aligned the finger 1 maps from the three experiments (Fig. 8, top left). The size of this patch was comparable with the areas of the individual finger domains. Also like many of the single-finger domains, the common activation in the anterior part of the gyrus was stronger than that in the posterior part.

As part of the confirmation of the optical data with single-unit and multiunit recordings in monkey $\mathrm{M} 3$, one of the penetrations to the common patch area was targeted. By using the same stimuli that were used for the imaging, we confirmed the imaging data, including the difference in response amplitude between stimulations of fingers 1 and 2 and stimulations of the other fingers. The multiunit tuning curve is shown in Figure $7 B$, left histogram, and the spike trains and PSTHs are shown in the bottom of Figure 8. These data confirmed the optical maps. Furthermore, they showed interesting differences in the temporal pattern of response. Although the total number of spikes in the responses to the stimulation of fingers 1 and 2 was similar (Fig. $7 B$ ), there was a clear difference in the distribution of these spikes in time (Fig. 8). The spikes evoked by finger 1 stimulation were more tightly locked to the stimulus pulses, and those evoked by finger 2 stimulation were more spread over the stimulus period. This is reflected also in the difference in the amplitude of the PSTHs. The responses to the other fingers (3-5) were not only weaker than that of fingers 1 and 2 but also different in the temporal pattern; the responses to the first few pulses were much more time-locked than were those to the last pulses.

In this penetration, after completing the recording of the response to the computer-controlled pneumatic stimuli, we also mapped the receptive field of the recorded units manually by lightly touching the skin at different locations. We found that the

penetration sites. The tuning curves obtained in each recording site are shown above as color-coded histograms. Thus despite the overlap, the responses are rather tuned. The vertical bars show the normalized spike counts from the $2 \mathrm{sec}$ stimulation period. The dashed horizontal lines show the spontaneous firing level that was computed from the periods with no stimulation. Note that the small inhibition mentioned above is apparent in all three recording sites that correspond to digits $1,2 / 3$, and 5. Each of the tuning curves was normalized to have a common maximal value. 


\section{Finger 1 Stimulation}
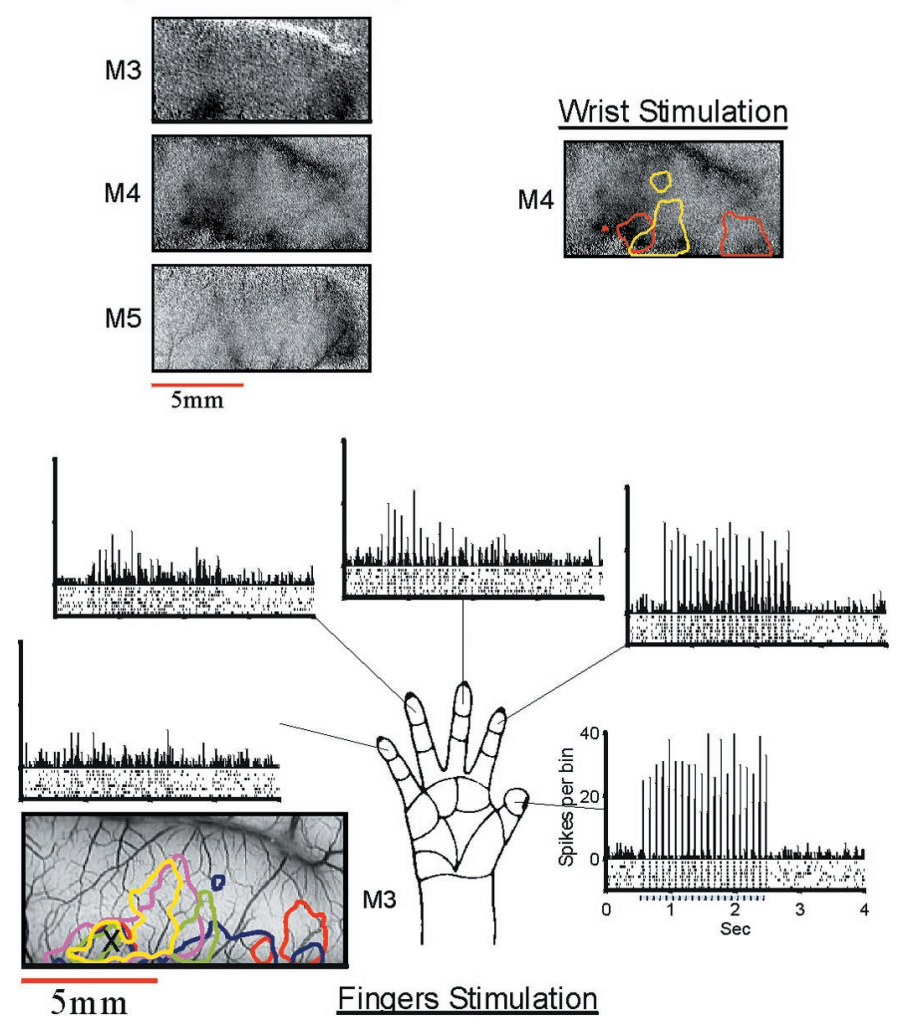

Figure 8. The common patch. Top left, The three images show the cortical activation by stimulation of the thumb in three monkeys. In addition to the finger-specific domain on the lateral (right) side, there is another activated patch on the medial part (left part of the image). This common patch was activated also by stimulation of the other fingers (data not shown). Top right, The map (monkey $M 4$ ) shows the activity evoked by stimulation of the wrist using the same pneumatic stimulator that was used for the fingers. This activation pattern also includes the common patch. To assist in comparison with the fingers somatotopy, the contour lines of fingers 1-5 from Figure 4 were superimposed on the map. The full gray scale corresponds to a fractional change of $1 \times 10^{-3}, 1.4 \times 10^{-3}$, and $1.1 \times 10^{-3}$ in the finger 1 images and to $1.1 \times 10^{-3}$ in the wrist image. Bottom, The multiunit data recorded at the common patch of monkey M3 are shown. The format is the same as that used in Figure $7 A$. The tuning curve is shown in Figure $7 B$.

units in this recording site were strongly activated by slight touch of all parts of the hand, the wrist, or the forearm and also weakly activated by upper arm stimulation.

In another experiment (M4), we attached a pneumatic stimulator, like those used to stimulate the fingers, to the wrist (approximately above the median nerve) and imaged the optical signals. The optical map shows that this stimulus also activated the common patch (Fig. 8, top right). However with the wrist stimulus, the activation was more uniform across the gyrus, unlike the activation caused by stimulating the fingers. In addition the posterior part of the gyrus (top of the image; presumably area 2) was activated almost to the same level as the anterior part (area 1).

\section{Median nerve stimulation}

At the first stage of this study, we mapped the cortical response to an electrical stimulation of the median nerve at the wrist. This was an effective stimulus that resulted in localized activation in the somatosensory cortex ( $\sim 10 \mathrm{~mm}$ along the gyrus). In those experiments we could not examine the correspondence of median nerve activation to the finger maps because we did not map the
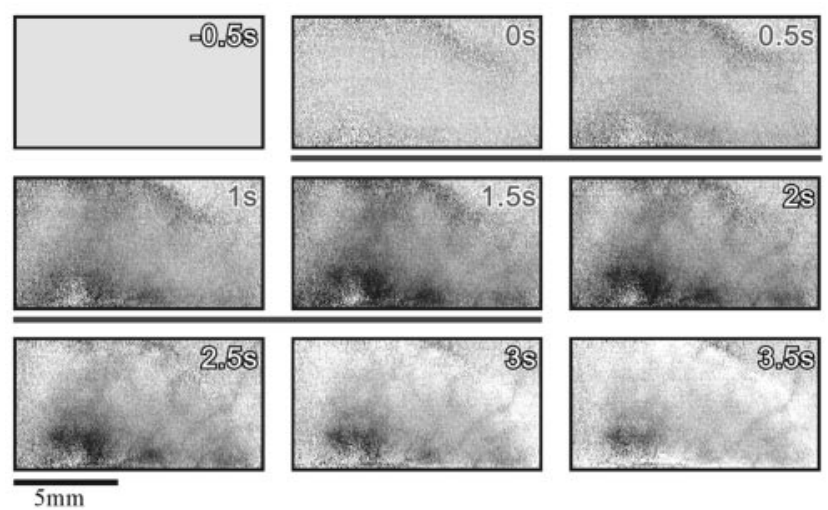

Figure 9. Time course of optical response to electrical stimulation of the median nerve. An image series shows the temporal development of the optical response to a right median nerve stimulation in monkey M4. Each frame represents $500 \mathrm{msec}$. The median nerve was stimulated at the wrist via a pair of EKG electrodes. Current pulses of $1 \mathrm{msec}$ were delivered for $2 \mathrm{sec}$ at a rate of $10 \mathrm{~Hz}$. The stimulus started concurrently with the acquisition of the second frame (stimulus duration is marked by a black horizontal bar). The full gray scale corresponds to a fractional change of $1.5 \times 10^{-3}$.

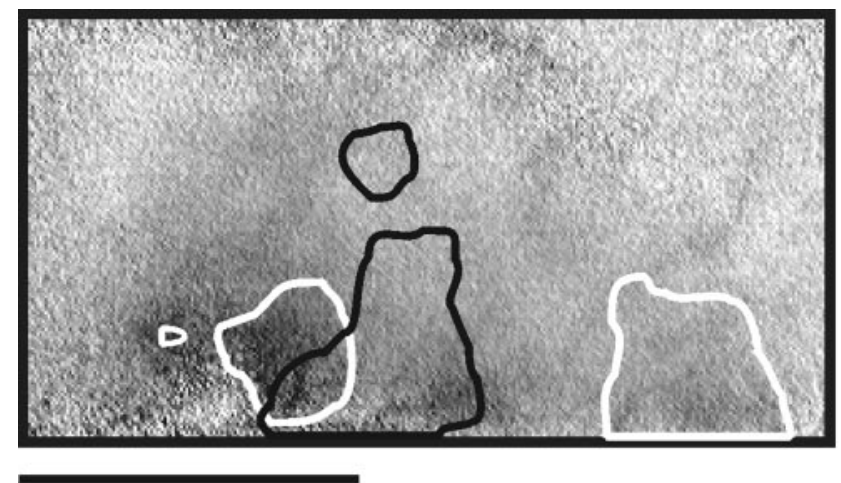

\section{$5 \mathrm{~mm}$}

Figure 10. Cortical activation by electrical stimulation of the median nerve. An activity map produced by averaging the last six frames from Figure 9 (corresponding to the period $1-4 \mathrm{sec}$ after stimulus onset) is shown. The contour lines of fingers 1 and 5 from Figure 4 are superimposed on the map to aid in comparison with the fingers somatotopy. Note that a much larger area was activated (light gray area darker than the top of the image), but the common patch was the strongest area activated and occupied much of the dynamic range of this figure. Note that median nerve stimulation in different experiments on different monkeys or human subjects often yields highly variable results, presumably because of the difficulty in reproducing exactly the same activation of the nerve in different subjects (data not shown). The full gray scale corresponds to a fractional change of $1.5 \times 10^{-3}$.

individual fingers. In one of the latest experiments, when we also mechanically stimulated individual fingers, we could compare the activation from the two types of stimuli in the same cortex. The time course of cortical activation resulting from the median nerve stimulation is shown in Figure 9. The amplitude of the optical signal was similar to that of the signal evoked by the tactile stimulus. The pattern of cortical activation resulting from the electrical stimulus is shown in Figure 10, in which the images obtained in the period 1-4 sec after stimulus onset were integrated. The contour lines from the finger maps are superimposed on the image. The comparison of the median nerve map with the finger maps shows that in this case the strongest activation was in 

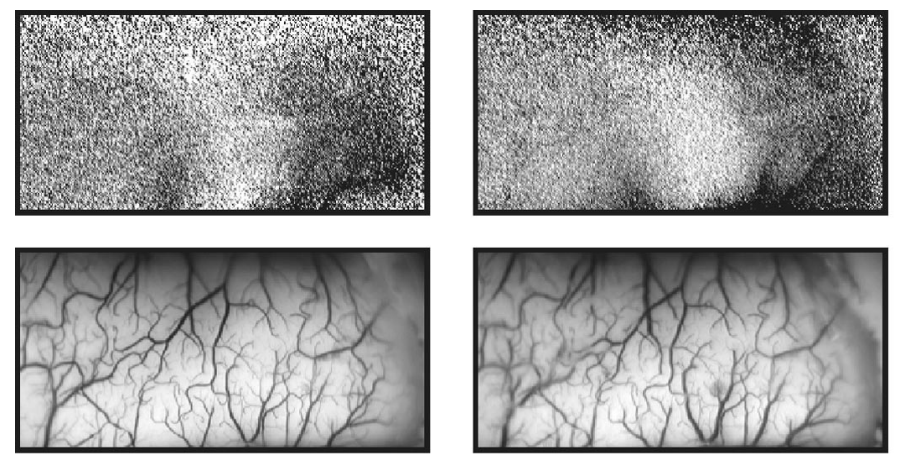

\section{$5 \mathrm{~mm}$}

Figure 11. Stability of cortical maps. Top, The images are differential maps in which the image obtained during simultaneous stimulation of fingers 1 and 2 was divided by the image from stimulation of fingers 3-5. The data for the left map were collected at the beginning of the experiment, and the map on the right was obtained $\sim 28 \mathrm{hr}$ later (in $28 \mathrm{hr}, \sim 50$ different maps can be accumulated under different stimulation conditions). The full gray scale corresponds to a fractional change of $0.8 \times 10^{-3}$ (left) and $1.4 \times 10^{-3}$ (right). Bottom, The images show the corresponding vascular patterns taken with green light.

the common patch area, medial to the hand representation. The pattern is quite similar to that evoked by a tactile stimulation of the wrist at the same site (see Fig. 8, top right). This suggests that some of the activation we saw after electrical stimulation in this case did not result from stimulation of the medial nerve but rather came from direct stimulation of the receptors at the wrist below the electrodes.

\section{Stability of the maps}

Some experimental questions require repeated imaging over many hours or days or imaging of the same cortical area before and after a time-consuming experimental manipulation. It is then important to verify the stability of the maps over many hours. To assess the stability of the optically imaged somatotopic maps in our preparation, we compared the maps obtained at the early stage of the experiment with those obtained toward the end, $\sim 28$ hr later. Figure 11 shows that the maps were very stable over this period. The maps shown here are differential maps obtained by simultaneously stimulating either fingers 1 and 2 or fingers 3-5. We often used this pair of stimuli in the beginning of the experiment, because it allowed us to delineate the region of interest and to assess the quality of the recording very quickly.

\section{Rapid functional imaging}

Because the optical signals are small and susceptible to various noise sources, extensive averaging is usually needed to obtain high-quality maps. There are, however, situations in which the recording time is limited and does not allow much averaging. In such situations it is crucial to be able to obtain at least an approximate version of the functional maps very quickly. In the current series of experiments, under optimal conditions for the animal, the functional maps could be obtained extremely rapidly. In the best recordings an experienced observer could detect the map even after a single trial. A clear map was often visible after a single block of only a few trials. This is demonstrated in Figure 12. The maps on the right were obtained by averaging the responses to only five stimulus presentations (and five blanks). A comparison of these maps with those obtained with more exten-

\section{TRIALS}
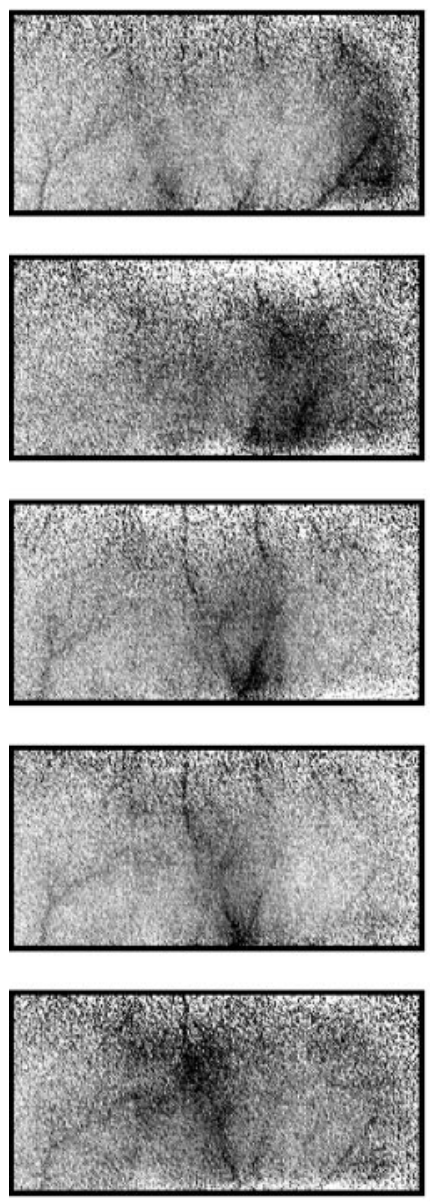

$5 \mathrm{~mm}$

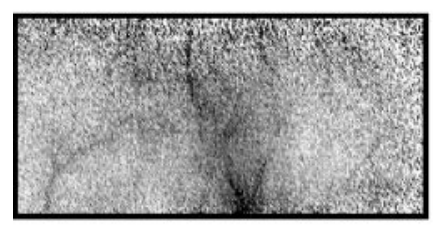

5 TRIALS
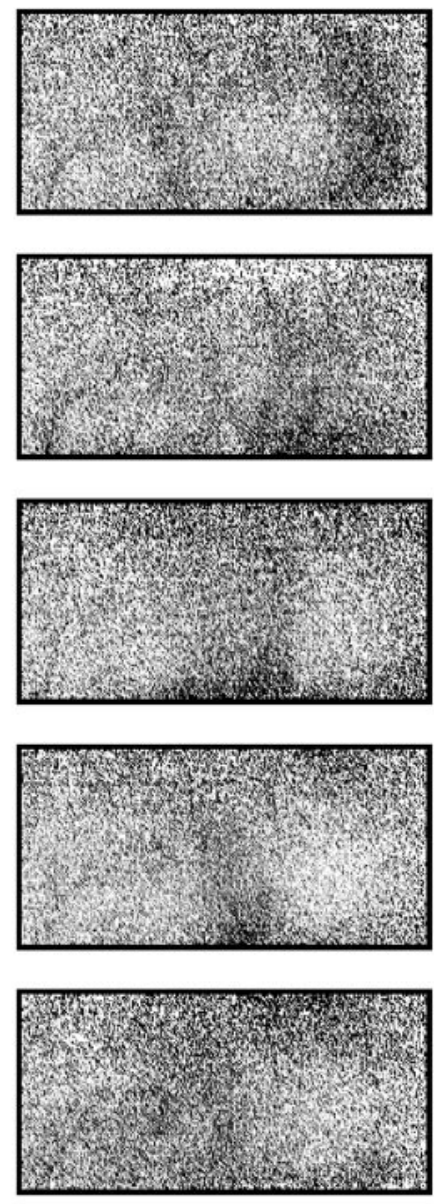

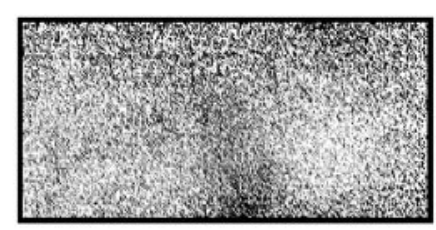

Figure 12. Rapid functional imaging. Single-finger maps from monkey M5, with finger 1 at the top and finger 5 at the bottom. Right, The images are from a single block of five trials. Left, The corresponding images are averages over 11 such blocks. The data collection for these maps took $\sim 6$ min per block. All images were autoclipped to occupy the full dynamic range of each image.

sive averaging (left) shows that the functional borders are quite visible with so little averaging. Because we presented approximately five stimuli per minute, the imaging of all five finger maps took only $\sim 6 \mathrm{~min}$. The complete data set (55 trials) was obtained in a little over an hour.

Having optimized the technique to such an extent, we attempted to apply this methodology in the operating room (OR) to assist neurosurgeons in delineating functional borders in the human brain before surgical procedures.

\section{Intraoperative optical imaging of human patients}

We used optical imaging intraoperatively in 15 patients undergoing tumor or AVM resection. In most of these patients (13), the surgery involved exposing the central region of the cerebrum, close to the sensorimotor areas. Many imaging sessions were used to adapt the imaging setup and procedures to the special needs of the intraoperative use. In some of these initial studies, the noise level was so high that it was expected to mask any evoked signals 


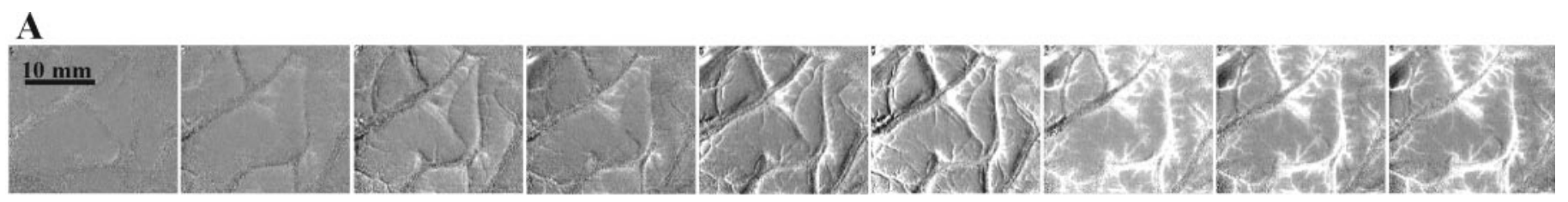

B

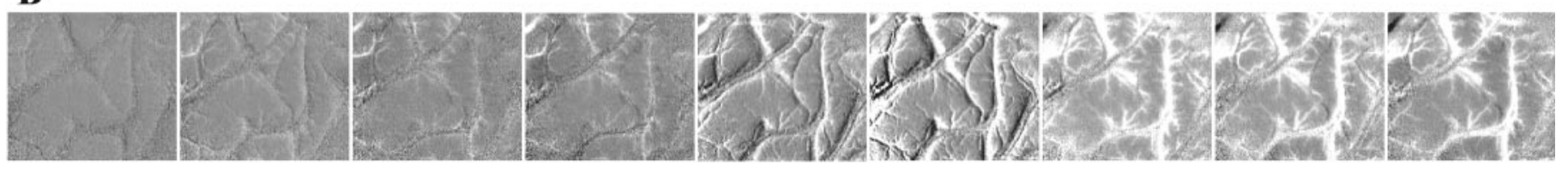

C
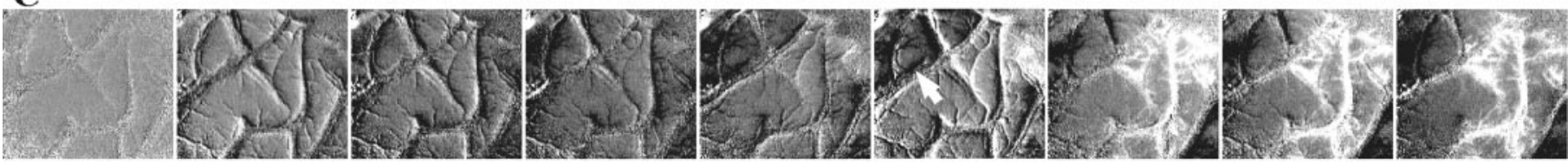

D
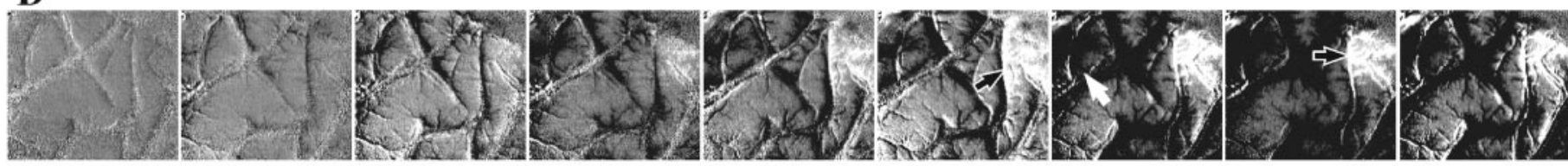

$\mathbf{E}$
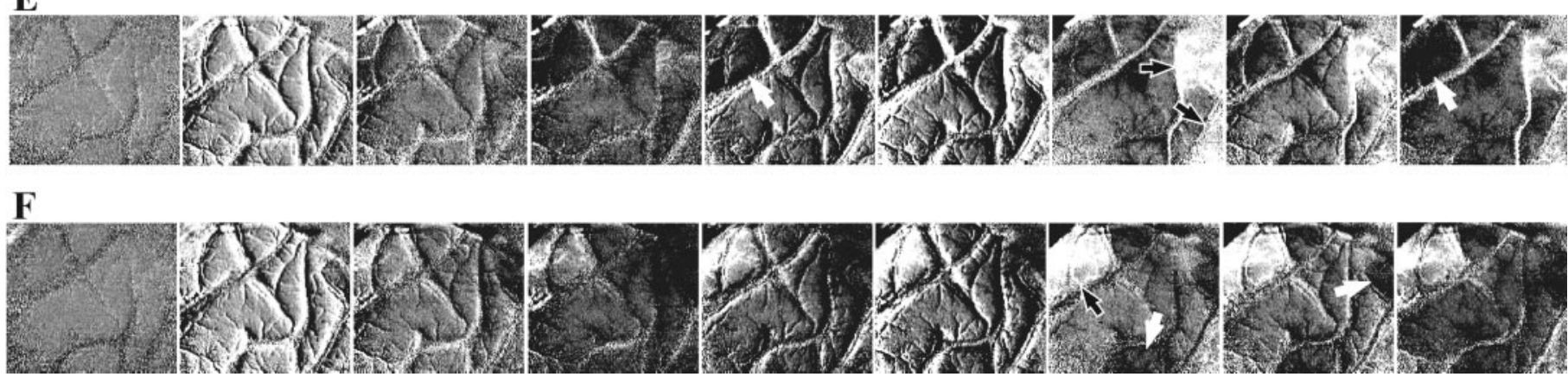

Figure 13. Visualization of spontaneous vascular activity in the human cortex. Each row shows spontaneous changes (without a stimulus) in cortical reflection as a function of time. Frame duration is $500 \mathrm{msec} . A, B$, These rows depict an increase in vascular oxygenation level (vessels brighten) over a large cortical area. The full gray scale (clipping range) corresponds to a large fractional change of $2.0 \times 10^{-2}$. These two similar events occurred 15 min apart. $C$, This row shows vascular activation as well as darkening of the parenchyma in a restricted area (white arrow). $D-F$, These rows depict slow brightening (black arrows) and darkening (white arrows) in a restricted area (clipping range, $2.0 \times 10^{-3}$ ). To show these small changes, these sequences of cortical images were obtained by subtracting the average of the first three frames in the sequence from each of the cortical images. Images were obtained with $605 \mathrm{~nm}$ illumination emphasizing oxygenation changes. Scale bar, $10 \mathrm{~mm}$.

of the amplitude found in the present monkey studies. By optimizing various aspects of the imaging apparatus and procedures as described in Materials and Methods, we managed to reduce the noise to a level that should allow clear visualization of the expected evoked optical signals. In some of these relatively lownoise imaging sessions, we thought that we detected evoked signals. On closer inspection, however, we found that the exact pattern of these responses varied from trial to trial even if the same stimulus was used. Thus, in all cases except one, we were not able to exclude that these responses were contaminated by vascular activity rather than neuronal activity. We concluded that it is important to understand the nature of the spontaneous vascular activity in anesthetized human subjects before optimal, noisefree, and reproducible functional maps can be readily obtained. Therefore, we started exploring the behavior of the cortical microcirculation without a sensory stimulus.

\section{Spontaneous fluctuations in blood flow/volume in anesthetized patients}

Here we focus on one case we studied extensively, although similar spontaneous fluctuations in flow were seen in most subjects. This paragraph describes the behavior of spontaneous vascular activity. The next paragraph then shows optical imaging of the hand representation of this subject, obtained by signal averaging, which was subsequently confirmed by electrical mapping. The case we report involved a 44-year-old male with a low-grade glioma in the left hemisphere that was estimated to be closely posterior to the somatosensory cortex. In a single-trial recording of $6.5 \mathrm{sec}$ sessions, we observed two different types of spontaneous vascular activity. First, slow changes in the oxygenation levels were readily seen in the large vessels as well as the parenchyma (e.g., Fig. 13A-C). Second, these vascular changes were often 

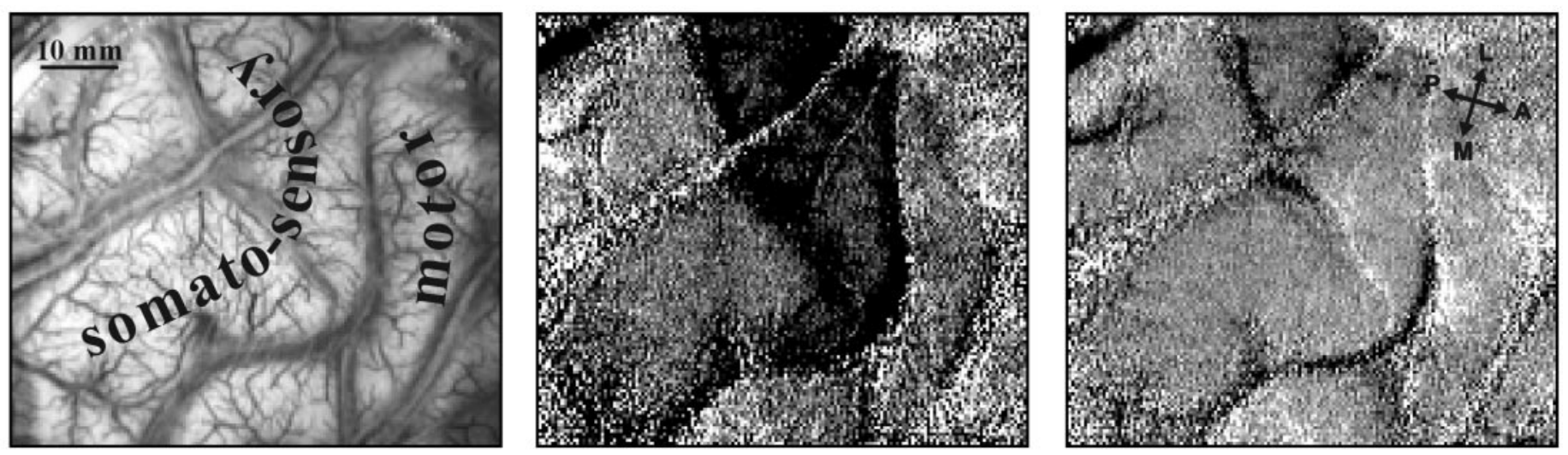

Figure 14. Intraoperative imaging of the somatosensory hand area using median nerve stimulation. Left, Image of the cortical surface (left hemisphere) illuminated with green light $(540 \mathrm{~nm})$ to emphasize the blood vessel pattern. The somatosensory and motor stripes as determined by optical imaging and confirmed with an electrocorticogram (see Fig. 15) are marked. Middle, Optical map of the area activated by median nerve stimulation. To minimize the noise, trials showing large vascular noise (such as that seen in Fig. 13A,B) above a fixed preset value were autorejected by the analysis program. To obtain this functional map, the average image (16 trials) from the stimulated conditions was divided by the average image from the blank condition (no stimulus, 16 trials). Right, Flat map showing the control map obtained by dividing the sum of the same blank condition by another independent set of the second blank condition we used as a control (16 trials each).

followed by a considerable darkening (Fig. $13 C-F$, white arrows) or whitening (Fig. 13D-F, black arrows) of the entire parenchyma in much smaller and well defined cortical areas, presumably "serviced" by a defined set(s) of an artery and veins. This vascular activity is presumably related to the well known vasomotion fluctuations (Schiff, 1854; Mayhew et al., 1996). However, it did not appear regular in each site. Thus, its exact temporal behavior remains to be explored. It is evident from the cases depicted in Figure 13 that such signals could be mistaken for functional maps, because they involved darkening or brightening of the cortical parenchyma and not just the larger blood vessels. Similar phenomena were observed in other patients and cannot be attributed to an anomalous cortical state of the patient.

\section{Imaging the human hand representation and its electrical confirmation}

Obviously, the very large fluctuations can be automatically rejected by preset criteria. The smaller type of noise (e.g., Fig. $13 D, E)$ could be minimized with signal averaging. In this patient we imaged optical- and electrical-evoked responses using an electrical stimulation of the median nerve at the wrist. This stimulus produced larger evoked activity then did tactile stimulation and should reveal the entire hand representation as shown in Figure 14. The left panel of Figure 14 shows the imaged cortical region with the motor and sensory strips marked as identified by the imaging and by the electrical recording. The tumor was just posterior to the imaged area. The optical map of the intrinsic signals evoked by the median nerve stimulation is shown in the middle panel of Figure 14, averaging over 16 repetitions that were summed and divided by the average image from the no-stimulus condition (also 16 repetitions). The dark triangle-like shape at the top is the somatosensory hand area. The right panel in Figure 14 shows the flat control map obtained by comparing two sets of no-stimulus conditions (16 repetitions in each). Evidently the noise was averaged out.

Stimulation of individual fingers always resulted in much weaker maps in the same area. Because of the current signal-tonoise ratio, we were unable to localize the individual finger representation within the general area of the hand, in a reproducible manner.
Figure 14 also demonstrates some of the problems associated with trying to estimate visually the location of even the major sulci during surgery; because of the small amount of cortex exposed, it was difficult to determine the identity of the sulci seen. Furthermore, in this case it was impossible to determine even the course of the sulci in the exposed area, because they were hidden below large blood vessels. Looking at the cortex in this particular case, we suspected that the large blood vessel, which runs diagonally at the top left of the image, parallels the course of a sulcus. As the imaging data showed, this sulcus (identified by the imaging as the postcentral sulcus) coincides with the blood vessel only in the bottom of the image, and where the blood vessel continues along a straight line across the postcentral gyrus, the sulcus takes a left turn.

To confirm the optical map, we used the well established method of recording SEP from the cortical surface. We used an array of $4 \times 5$ electrodes (Fig. 15, left) and recorded each time from a selected group of 8 electrodes simultaneously. The results from two such recordings are shown in Figure 15, right. The electrodes from which these recordings were done are colored yellow on the photographic image of the brain (Fig. 15, left). The median nerve stimulation (Fig. 15, right, top) resulted in a large signal over a large cortical area nearly coinciding with the area that was identified as the hand area in the imaging session. The tactile stimulation of a single finger resulted in the SEP signal, which had a lower amplitude than did the median nerve stimulation (note the calibration bars) but was much more localized, almost limited to a single electrode (labeled by a green circle; Fig. 15). These results indicate that the resolution of the surfaceevoked potential is better than traditionally believed. It is difficult to assess whether the pioneering OR studies by optical imaging offered a better signal-to-noise ratio. In our hands it appears that further improvements are needed before high-resolution optical imaging can be obtained from anesthetized patients.

\section{DISCUSSION}

We have obtained reproducible somatotopic maps of the hand representations in both Brodmann's areas 1 and 2. The responses to the tactile stimuli were generally weaker in area 2 than in area 


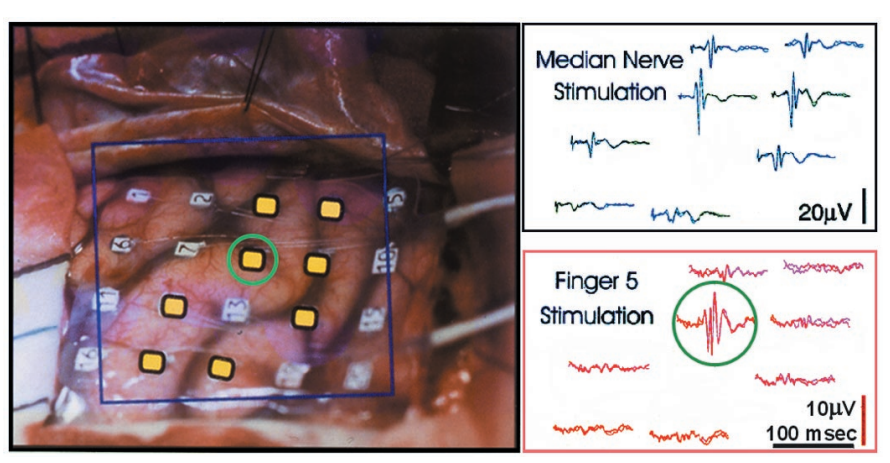

Figure 15. Electrocorticographic confirmation of the optical map. Left, A photographic image of the exposed cortex with the array of surface electrodes $(4 \times 5)$ that were used to record somatosensory-evoked potentials (boxed area). Right, Electrical traces showing the evoked potentials that were recorded simultaneously from eight electrodes (colored yellow on the photo). The arrangement of the traces mirrors the position of the corresponding electrodes (see Materials and Methods). The top set of traces (blue) shows responses to electrical stimulation of the median nerve, and the bottom traces (red) show the response to tactile stimulation to the tip of finger 5. Each trace is actually a composite, consisting of two superimposed traces representing the average from two independent sets of trials (128 repetitions for the median nerve and 256 repetitions for the finger 5 stimulation).

1. The optical maps were confirmed by targeted unit recordings. We have characterized the spatiotemporal patterns of different components of the intrinsic optical signals. In addition to the evoked signals, we have found slow high-amplitude fluctuations in cortical reflectance that could drastically distort the functional maps in the monkey and human brain, particularly under anesthesia. The results of the experiments described here are also relevant to general methodological issues, particularly to the spatial resolution of functional brain mapping using metabolic signals such as optical imaging, positron emission tomography (PET), and f-MRI. We will discuss this topic first.

\section{The hemodynamic signals: implications to other imaging modalities}

In this study we imaged a relatively large area that included two major sulci (the central and the intraparietal sulci) and the large blood vessels that run along these sulci. Examination of the temporal development of the evoked optical signals in different parts of the imaged area revealed two major response components: a fast, localized, stimulus-specific darkening and a slow nonspecific brightening (Figs. $1 B, 2 B$ ). Regardless of the stimulus used, the brightening was evident over the whole imaged area (which included not only nonhand somatosensory cortex but also part of the two adjacent giri), and it was highest in the large vessels (which do not pass directly over the somatotopically activated area). Previous studies of the sources of the intrinsic optical signals (Grinvald et al., 1986, 2000; Frostig et al., 1990; Malonek and Grinvald, 1996) suggest that the localized component is mainly caused by an increase in oxygen consumption, light scattering, and blood volume at the active area and that the late brightening is caused by increased blood flow, which elevates the oxyhemoglobin/deoxyhemoglobin ratio. To optimize the quality of the high-resolution somatotopic maps, we used the procedure that has become standard in optical imaging, namely, to present short stimuli ( $\sim 2 \mathrm{sec})$ with longer interstimulus intervals (7-15 sec) and to use only the images from the first few seconds of the response. As was discussed previously (Malonek and Grinvald, 1996; Vanzetta and Grinvald, 1999, 2001; Grinvald et al., 2000), the optical analysis of the cortical microcirculation signals has clear implications to other modern imaging techniques, such as PET and f-MRI, which also measure blood-related signals. The current study further emphasizes these implications. PET is known to measure blood-flow changes. Most f-MRI BOLD studies are also thought to rely on these changes by imaging the decrease in deoxyhemoglobin during the increased flow phase (Kwong et al., 1992; Ogawa et al., 1992). The relatively slow time course (time to peak, $\sim 4-8 \mathrm{sec}$ ) and the polarity of most reported f-MRI signals (positive signals, supposedly reflecting an increase of oxyhemoglobin) suggest that their sources might be similar to some of the slow, nonlocal brightening that we saw in the optical imaging. The spatial characteristics and the nonspecificity of the presumed blood-flow component that was shown here imply that relying on the blood-flow signals alone should severely limit the spatial resolution that may be obtained. This limitation, hopefully, can be removed by developing procedures that boost the local components of the intrinsic signals (e.g., by using higher magnetic fields or different f-MRI sequences, emphasizing the signals of capillaries, etc.) and/or by using stimulus presentation and analysis procedures more similar to those used in optical imaging (Grinvald et al., 2000; Vanzetta and Grinvald, 2001). First steps in these directions have been reported previously, yet they remained controversial (Menon et al., 1995, 1997; Hu et al., 1997; Logothetis et al., 1999; Yacoub et al., 1999; Kim et al., 2000a,b; Logothetis, 2000). Besides the issue of the evoked signals, attention should also be paid to the possibility that the very large slow spontaneous fluctuations that were discussed above (e.g., Fig. 13) might also contaminate maps obtained by other neuroimaging techniques based on and/or affected by responses of the cortical microcirculation. There have been several reports of positive and negative changes in reflectance of large cortical areas during cognitive tasks, which were interpreted as reflecting the pattern of neuronal excitation and inhibition (Hochman et al. 1994). Such observations were made with optical imaging, PET, and functional MRI, all relying on hemodynamic signals. Our finding of very similar events in the absence of a stimulus (Fig. 13) casts some doubt on this interpretation. We had hoped that these fluctuations that may severely hamper spatial resolution are apparent only in the anesthetized monkey and human and thus are not relevant to awake human brain mapping. Therefore, we reexamined the presence of such slow fluctuations also in the awake monkey. We found them to be similar yet of a smaller amplitude (I. Vanzetta, H. Slovin, and A. Grinvald, unpublished observations). It appears, therefore, that comparative studies in awake macaque monkeys, similar to those described here, should accelerate the improvements of promising approaches for noninvasive imaging including fast optical brain imaging through the intact human skull (Gratton and Fabiani, 1998).

\section{Functional organization of the hand representation in areas 1 and 2}

The imaging results presented in this paper corroborate the known somatotopic organization of the hand area as studied previously with multiunit recordings in area 1 (Nelson et al., 1980) and area 2 (Pons et al., 1985) of the macaque monkey. In both areas, the fingers were represented sequentially, proceeding from the thumb to the little finger in a lateromedial sequence. The finger sequences in the anterior and posterior parts of the gyrus (presumably corresponding to areas 1 and 2) primarily paralleled each other with some deviations. Also in agreement with the previously reported maps, we found that the thumb had the 
largest territory and fingers 3-5 had the smallest. Nevertheless, all stimuli activated areas of approximately the same size, but with larger overlap between fingers 3, 4, and 5. The responses we recorded were stronger in the anterior part of the gyrus, and sometimes there was even a gap between the anterior and the posterior activations. This suggests that although we cannot be certain about the exact location of the border between the two areas, this border might be reflected in the present functional maps. It appears from our results that area 2 is less responsive to the tactile stimuli we used than is area 1 . This is probably true only for simple punctate stimuli in the anesthetized preparation. Previous studies in behaving monkeys suggested that neurons in area 2 are driven best by complex stimuli during active touch, when the monkey is touching a textured surface or grasping an object (Iwamura and Tanaka, 1978; Costanzo and Gardner, 1980; Gardner and Costanzo, 1980; Darian-Smith et al., 1984, 1985; Iwamura et al., 1985b, 1995). It should be very interesting to image the patterns of activity in area 2 during such behaviors. In the current study we focused on the somatotopic map, neglecting other aspects of the functional architecture such as the columnar organization of the different submodalities that has been described in area 3b (Paul et al., 1972). Thus similar imaging experiments are desirable, in which the same skin locations are stimulated using different kinds of well defined stimuli that would differentially activate each receptor type.

\section{The common patch}

When we first imaged the cortical response to the tactile stimuli, we were surprised to find that the stimulation of the fingers evoked activity, not only in the expected somatotopic position within the hand area, but also in the adjacent common patch of cortex, just medially to the fingers representation. This result was then replicated in the other two monkeys and was confirmed by unit recordings. According to previous mapping studies, this area represents the ulnar volar pads (Nelson et al., 1980; Pons et al., 1985). An obvious possible explanation for the common patch we found is that the stimuli used were perhaps too strong and therefore activated receptors in the ulnar pads as well. However, such an explanation was eliminated. In all three animals, stimulating fingers 1 and 2 activated this patch more than did stimulating the ulnar fingers. If stimulation 1 and 2 indirectly activated receptors in the ulnar pads, we would expect an even stronger activation in the radial pads, which would show up as an activation in the cortical area lateral to the finger representation (Nelson et al., 1980; Pons et al., 1985). No such activation was seen. The very large size of the receptive fields found in the electrical recording casts further doubt on this explanation.

We have shown that the common patch was activated by even fairly weak stimulation of a very large skin area, which includes the entire hand, wrist, and forearm, and to a smaller degree by the upper arm too. Other researchers observed similar receptive fields in area 1 of the macaque (Favorov and Whitsel, 1988a,b) (E. P. Gardner and R. M. Costanzo, personal communication). The large size of these receptive fields may suggest that the common patch may be dominated by input from the very sensitive Pacinian receptors. Indeed there are some reports of Pacinianlike responses in a small fraction (1-4\%) of the penetrations into the hand representation of area 1 (Paul et al., 1972; Hyvärinen and Poranen, 1978; Kaas et al., 1979; Iwamura et al., 1983b). It is unclear from these reports whether these responses are spatially organized in any way, although Paul et al. (1972; their Fig. 2) showed an example of Pacinian-like responses in three adjacent penetrations. Our findings show that these responses are confined to a particular patch of cortex, suggesting that a fine-grained somatotopic map does not represent the Pacinian receptors. To gain a better understanding of the functional significance of the common patch, further research is needed to clarify the relation between the common patch and Pacinian inputs and the particular response preferences of neurons in the common patch for fingers 1 and 2 .

\section{Overlapping representations?}

Traditional studies of the somatosensory cortex have mainly used threshold-level stimuli and the "minimal receptive field" (Merzenich et al., 1978) mapping approach in deeply anesthetized animals. These studies presented well defined somatotopic maps with discrete parcelation of the cortex into nonoverlapping zones, although some multidigit receptive fields were reported, mainly in area 2 (Pons et al., 1985). On the other hand, studies in awake behaving monkeys using mainly suprathreshold stimuli have shown more complex maps, with individual cells typically responding to multiple digits (Iwamura et al., 1983a,b, 1985a; Gardner, 1988; Favorov and Whitsel, 1988a,b). In some cases, especially in areas 1 and 2, somatotopy was not evident. The difference between the maps obtained in these two types of studies can be attributed to the difference between awake and anesthetized animals, to the difference between threshold and suprathreshold stimuli, or to some other methodological differences. The results reported here can shed light on this issue.

Our experiments were done in anesthetized animals using suprathreshold stimuli. Although anesthesia has been shown to reduce the apparent size of receptive fields (Duncan et al., 1982; McKenna et al., 1982), we nevertheless observed significant overlap between the activations evoked by neighboring fingers. Thus the presence or absence of overlap cannot be attributed solely to the differences in anesthesia. Could part of the overlap be attributed to methodological issues related to optical imaging of intrinsic signals?

Other optical imaging studies (which also used suprathreshold stimuli) have shown a similar representational overlap both in the somatosensory cortex of the rat (Masino et al., 1993; Godde et al., 1995) and in the auditory cortex of the rat, guinea pig, and gerbil (Bakin et al., 1996; Hess and Scheich, 1996). Because the origin of intrinsic signals is metabolic, they only indirectly reflect the neuronal electrical activity and are less localized than are the electrically active area. At the wavelength we used $(605 \mathrm{~nm})$, the signal spread is relatively small but still exists. Some blurring can also result from light scattering in the cortical tissue and from out-offocus contributions. Because all of these studies produced true single-condition maps, the blurring effects were not canceled out as they are in the commonly used differential maps. However the spread produced by light scattering was estimated to be in the range of 200-300 $\mu \mathrm{m}$ (Orbach and Cohen, 1983) and, therefore, cannot fully account for the much larger overlap seen here.

The nature of intrinsic signals cannot, however, account for the apparent overlap, because we found overlap also in the multiunit recordings (Fig. $7 B$ ). Similar correlations between spike activity and optical signals have also been shown in the rat and guinea pig somatosensory and auditory cortices (Masino et al., 1993; Bakin et al., 1996). Thus our results demonstrate that the overlap in the representations of the different fingers can be revealed by using suprathreshold stimuli, even under anesthesia. Clearly, the perception of suprathreshold tactile stimuli, which are common in a normal environment, is supported by activity in large neuronal 
populations, which go beyond the somatotopic representations of subthreshold stimuli that evoke subthreshold synaptic activation in the cortex, not readily revealed by single-unit recordings. Because of the present data and the activation patterns in S-I seen in response to digit tip activation, reported in previous $2 \mathrm{DG}$ studies (Juliano et al., 1981; Juliano and Whitsel, 1985), we believe that the significant overlap we observed is functionally significant and has general implications for columnar organization of the S-I area. However, we do not know which fraction of the overlap that we have seen should be attributed to subthreshold synaptic activation reflected by intrinsic imaging (Das and Gilbert, 1995, 1997) or to spiking activity.

\section{Intraoperative optical imaging}

In the case presented here it was impossible to identify visually even the central sulcus, intraoperatively. After the optical imaging and its confirmation by SEP (Goldring and Gregorie, 1984; Gregorie and Goldring, 1984; Wood et al., 1988), the resection was performed avoiding this region, and when the patient recovered, he had full motor control and sensation in his right hand. This case as well as a similar previous report by Cannestra et al. (1998) demonstrates the potential use of intraoperative optical imaging to provide the much-needed detailed functional maps of the operated area. However, to realize this potential for a broader use, additional improvement must be introduced to overcome the relatively large vascular noise and motion artifacts found under these conditions. One possibility to improve the quality of the optical imaging is to use longer wavelength for illumination, thus emphasizing the light-scattering signal, which is smaller but to a large extent does not reflect the hemodynamic signals that were imaged in this study. Another possibility is to correct some of the motion artifact by reregistration of the images, a method introduced by Cannestra et al. (1998).

\section{Concluding remarks}

The results presented in this paper demonstrate the ability of optical imaging based on an intrinsic signal to provide highresolution spatial maps of the primate somatosensory cortex in vivo. This opens a wide range of possibilities for further studies of the structure and function of this cortical region. The stability and reproducibility of the optical maps over time suggest that it should also be possible to use optical imaging to study long-term plastic changes in the primate somatosensory system. As we have shown here, using optimized stimulation and data analysis procedures, it is possible to obtain a picture of the functional architecture very rapidly. This should facilitate complementary explorations by using optical imaging to guide targeted unit recordings, tracer injection, microstimulation, etc. The rapidity of mapping also suggests that this technique is potentially valuable for intraoperative mapping of the human brain. However, comparing the map quality we obtained from monkey and human brain suggests that further efforts are needed before optical imaging can be used routinely to assist neurosurgery. After such improvements, a more widespread use of intraoperative optical imaging should lead to significant clinical advances and in addition would provide the highest resolution maps of functional representations in the human brain.

\section{REFERENCES}

Bakin JS, Kwon MC, Masino SA, Weinberger NM, Frostig RD (1996) Suprathreshold auditory cortex activation visualized by intrinsic signal optical imaging. Cereb Cortex 6:120-130.

Bonhoeffer T, Grinvald A (1993) The layout of iso-orientation domains in area 18 of cat visual cortex: optical imaging reveals a pinwheel-like organization. J Neurosci 13:4157-4180.
Bonhoeffer T, Kim DS, Malonek D, Shoham D, Grinvald A (1995) Optical imaging of the layout of functional domains in area 17 and across the area $17 / 18$ border in cat visual cortex. Eur J Neurosci 7:1973-1988.

Buonomano DV, Merzenich MM (1998) Cortical plasticity: from synapses to maps. Annu Rev Neurosci 21:149-186.

Cannestra AF, Black KL, Martin NA, Cloughesy T, Burton JS, Rubinstein E, Woods RP, Toga AW (1998) Topographical and temporal specificity of human intraoperative optical intrinsic signals. NeuroReport 9: 2557-2563.

Chapman B, Stryker MP, Bonhoeffer T (1996) Development of orientation preference maps in ferret primary visual cortex. J Neurosci 16:6443-6453.

Chen-Bee CH, Kwon MC, Masino SA, Frostig RD (1996) Areal extent quantification of functional representations using intrinsic signal optical imaging. J Neurosci Methods 68:27-37.

Costanzo RM, Gardner EP (1980) A quantitative analysis of responses of direction-selective neurons in somatosensory cortex of awake monkeys. J Neurophysiol 43:1319-1341.

Darian-Smith I, Goodwin A, Sugitani M, Heywood J (1984) The tangible features of textured surfaces: their representation in the monkey's somatosensory cortex. In: Dynamic aspects of neocortical function (Edelman G, Gall WE, Cowan WM, eds), pp 475-500. New York: Wiley.

Darian-Smith I, Goodwin A, Sugitani M, Heywood J (1985) Scanning a textured surface with the fingers: events in sensorimotor cortex. Exp Brain Res [Suppl] 10:17-43.

Das A, Gilbert CD (1995) Long-range horizontal connections and their role in cortical reorganization revealed by optical recording of cat primary visual cortex. Nature 375:780-784.

Das A, Gilbert CD (1997) Distortions of visuotopic map match orientation singularities in primary visual cortex. Nature 387:594-598.

Duncan GH, Dreyer DA, McKenna TM, Whitsel BL (1982) Dose- and time-dependent effects of ketamine on SI neurons with cutaneous receptive fields. J Neurophysiol 47:677-699.

Favorov O, Whitsel BL (1988a) Spatial organization of the peripheral input to area 1 cell columns. I. The detection of "segregates." Brain Res Rev 13:25-42.

Favorov O, Whitsel BL (1988b) Spatial organization of the peripheral input to area 1 cell columns. II. The forelimb representation achieved by a mosaic of segregates. Brain Res Rev 13:43-56.

Frostig RD, Lieke EE, Ts'o DY, Grinvald A (1990) Cortical functional architecture and local coupling between neuronal activity and the microcirculation revealed by in vivo high-resolution optical imaging of intrinsic signals. Proc Natl Acad Sci USA 87:6082-6086.

Gardner EP (1988) Somatosensory cortical mechanisms of feature detection in tactile and kinesthetic discrimination. Can J Physiol Pharmacol 66:439-454

Gardner EP, Costanzo RM (1980) Neuronal mechanisms underlying direction sensitivity of somatosensory cortical neurons in awake monkeys. J Neurophysiol 43:1342-1354.

Godde B, Hilger T, von Seelen W, Berkefeld T, Dinse HR (1995) Optical imaging of rat somatosensory cortex reveals representational overlap as topographic principle. NeuroReport 7:24-28.

Goldring S, Gregorie EM (1984) Surgical management of epilepsy using epidural recordings to localize the seizure focus. J Neurosurg 60:457-466.

Gratton G, Fabiani M (1998) Dynamic brain imaging: event-related optical signal (EROS) measures of the time course and localization of cognitive-related activity. Psychon Bull Rev 5:535-563.

Gregorie EM, Goldring S (1984) Localization of function in the excision of lesions from the sensorimotor region. J Neurosurg 61:1047-1054.

Grinvald A, Lieke E, Frostig RD, Gilbert CD, Wiesel TN (1986) Functional architecture of cortex revealed by optical imaging of intrinsic signals. Nature 324:361-364.

Grinvald A, Malonek D, Glaser D, Vanzetta I, Shtoyerman E, Shoham D (1999a) Intrinsic signal imaging in the neocortex. In: Imaging: a laboratory manual (Yuste R, Lanni F, Konnerth A, eds). Plainview, NY: Cold Spring Harbor Laboratory.

Grinvald A, Shoham D, Shmuel A, Glaser DE, Vanzetta I, Shtoyerman E, Slovin H, Wijnbergen C, Hildesheim R, Sterkin A, Arieli A (1999b) In-vivo optical imaging of cortical architecture and dynamics. In: Modern techniques in neuroscience research (Windhorst $\mathrm{U}$, Johansson $\mathrm{H}$, eds), pp 894-969. Heidelberg: Springer.

Grinvald A, Slovin H, Vanzetta I (2000) Non-invasive visualization of cortical columns by f-MRI. Nat Neurosci 3:105-107.

Haglund MM, Ojemann GA, Hochman DW (1992) Optical imaging of epileptiform and functional activity in human cerebral cortex. Nature 358:668-671.

Hess A, Scheich H (1996) Optical and FDG mapping of frequencyspecific activity in auditory cortex. NeuroReport 7:2643-2647.

Hochman DW, Ojemann GA, Haglund MM (1994) Optical imaging reveals alternating positive and negative changes during cognitive or sensory evoked cortical activity in awake humans. Soc Neurosci Abstr 20:9.1. 
Hu XP, Le TH, Ugurbil K (1997) Evaluation of the early response in fMRI in individual subjects using short stimulus duration. Magn Reson Med 37:877-884.

Hubel DH, Wiesel TN (1965) Receptive fields and functional architecture in two non-striate visual areas (18 and 19) of the cat. J Neurophysiol 28:229-289.

Hyvärinen J, Poranen A (1978) Receptive field integration and submodality convergence in the hand area of the post-central gyrus of the alert monkey. J Physiol (Lond) 283:539-556.

Iwamura Y, Tanaka M (1978) Postcentral neurons in hand region of area 2: their possible role in the form discrimination of tactile objects. Brain Res 150:662-666.

Iwamura Y, Tanaka M, Sakamoto M, Hikosaka O (1983a) Functional subdivision representing different finger regions in area 3 of the first somatosensory cortex of the conscious monkey. Exp Brain Res 51:315-326

Iwamura Y, Tanaka M, Sakamoto M, Hikosaka O (1983b) Converging patterns of finger representation and complex response properties of neurons in area 1 of the first somatosensory cortex of the conscious monkey. Exp Brain Res 51:327-337.

Iwamura Y, Tanaka M, Sakamoto M, Hikosaka O (1985a) Functional surface integration, submodality convergence, and tactile feature detection in area 2 of the monkey somatosensory cortex. Exp Brain Res [Suppl] 10:44-58.

Iwamura Y, Tanaka M, Sakamoto M, Hikosaka O (1985b) Diversity in receptive field properties of vertical neuronal arrays in the crown of the postcentral gyrus of the conscious monkey. Exp Brain Res 58:400-411.

Iwamura Y, Tanaka M, Hikosaka O, Sakamoto M (1995) Postcentral neurons of alert monkeys activated by the contact of the hand with objects other than the monkey's own body. Neurosci Lett 186:127-130.

Juliano S, Whitsel BL (1985) Metabolic labeling associated with index finger stimulation in monkey SI: between animal variability. Brain Res 342:242-251.

Juliano S, Hand P, Whitsel B (1981) Patterns of increased metabolic activity in somatosensory cortex of monkeys Macaca fascicularis, subjected to controlled cutaneous stimulation: a 2-deoxyglucose study. J Neurophysiol 46:1260-1284.

Kaas JH (1991) Plasticity of sensory and motor maps in adult mammals. Annu Rev Neurosci 14:137-167.

Kaas JH, Nelson RJ, Sur M, Lin CS, Merzenich MM (1979) Multiple representations of the body within the primary somatosensory cortex of primates. Science 204:521-523.

Kido D, LeMay M, Levinson A, Benson W (1980) Computed tomographic localization of the precentral gyrus. Radiology 135:373-377.

Kim DS, Bonhoeffer T (1994) Reverse occlusion leads to a precise restoration of orientation preference maps in visual cortex. Nature 370:370-372

Kim DS, Doung TQ, Kim SG (2000a) High-resolution mapping of isoorientation columns by fMRI. Nat Neurosci 3:164-199.

Kim DS, Doung TQ, Kim SG (2000b) Reply to "Can current fMRI techniques reveal the micro-architecture of cortex?" Nat Neurosci 3:414.

Kwong KK, Belliveau JW, Chesler DA, Goldberg IE, Weisskoff RM, Poncelet BP, Kennedy DN, Hoppel BE, Cohen MS, Turner R, Cheng H, Brady TJ, Rosen BR (1992) Dynamic magnetic resonance imaging of human brain activity during primary sensory stimulation. Proc Natl Acad Sci USA 89:5675-5679.

Logothetis NK (2000) Can current fMRI techniques reveal the microarchitecture of cortex? Nat Neurosci 3:413-414.

Logothetis NK, Guggenberger H, Peled S, Pauls J (1999) Functional imaging of the monkey brain. Nat Neurosci 2:555-562.

MacVicar BA, Hochman D, LeBlanc F, Watson T (1990) Stimulation evoked changes in intrinsic optical signals in the human brain. Soc Neurosci Abstr 16:138.12.

Malonek D, Grinvald A (1996) Interactions between electrical activity and cortical microcirculation revealed by imaging spectroscopy: implications for functional brain mapping. Science 272:551-554

Malonek D, Shoham D, Ratzlaff E, Grinvald A (1990) In vivo three dimensional optical imaging of functional architecture in primate visual cortex. Soc Neurosci Abstr 16:130.4.

Masino SA, Frostig RD (1996) Quantitative long-term imaging of the functional representation of a whisker in rat barrel cortex. Proc Natl Acad Sci USA 93:4942-4947.

Masino SA, Kwon MC, Dory Y, Frostig RD (1993) Characterization of functional organization within rat barrel cortex using intrinsic signal optical imaging through a thinned skull. Proc Natl Acad Sci USA 90:9998-10002

Mayhew JE, Askew S, Zheng Y, Porril J, Max Westby GW, Redgrave P, Rector DM, Harper RM (1996) Cerebral vasomotion: a 0.1-Hz oscillation in reflected light imaging of neural activity. NeuroImage 4:183-193.

McKenna TM, Whitsel BL, Dreyer DA (1982) Anterior parietal cortical topographic organization in macaque monkey: a reevaluation. J Neurophysiol 48:289-317.

Menon RS, Ogawa S, Hu X, Strupp JP, Anderson P, Ugurbil K (1995) BOLD based functional MRI at 4 tesla includes a capillary bed contri- bution: echo-planar imaging correlates with previous optical imaging using intrinsic signals. Magn Reson Med 33:453-459.

Menon RS, Ogawa S, Strupp JP, Ugurbil K (1997) Ocular dominance in human V1 demonstrated by functional magnetic resonance imaging. J Neurophysiol 77:2780-2787.

Merzenich MM, Kaas JH, Sur M, Lin CS (1978) Double representation of the body surface within cytoarchitectonic areas $3 \mathrm{~b}$ and 1 in "SI" in the owl monkey (Aotus trivirgatus). J Comp Neurol 181:41-73.

Mountcastle VB (1957) Modality and topographic properties of single neurons of cat's somatic sensory cortex. J Neurophysiol 20:408-434.

Nelson RJ, Sur M, Felleman DJ, Kaas JH (1980) Representations of the body surface in postcentral parietal cortex of Macaca fascicularis. J Comp Neurol 192:611-643.

Ogawa S, Tank DW, Menon R, Ellermann JM, Kim SG, Merkle H, Ugurbil K (1992) Intrinsic signal changes accompanying sensory stimulation: functional brain mapping with magnetic resonance imaging. Proc Natl Acad Sci USA 89:5951-5955.

Orbach HS, Cohen LB (1983) Optical monitoring of activity from many areas of the in vitro and in vivo salamander olfactory bulb: a new method for studying functional organization in the vertebrate CNS. J Neurosci 3:2251-2262.

Paul RL, Merzenich MM, Goodman H (1972) Representation of slowly and rapidly adapting cutaneous mechanoreceptors of the hand in Brodmann's areas 3 and 1 of Macaca mulatta. Brain Res 36:229-249.

Penfield W, Boldrey E (1937) Somatic motor and sensory representation in the cerebral cortex of man as studied by electrical stimulation. Brain 60:389-443.

Polley DB, Chen-be CH, Frostig RD (1999) Two directions of plasticity in the sensory deprived adult cortex. Neuron 24:623-637.

Pons TP, Garraghty PE, Cusick CG, Kaas JH (1985) The somatotopic organization of area 2 in macaque monkeys. J Comp Neurol 241:445-466.

Ratzlaff EH, Grinvald A (1991) A tandem-lens epifluorescence macroscope: hundred-fold brightness advantage for wide-field imaging. J Neurosci Methods 36:127-137.

Schiff L (1854) An accessory arterial heart in the rabbit (in German). Arch Physiol Heilk (Leipzig) 13:523-527.

Shoham D, Hubener M, Grinvald A, Bonhoeffer T (1997) Spatiotemporal frequency domains and their relationship to cytochrome oxidase staining in cat visual cortex. Nature 385:529-534.

Shtoyerman E, Arieli A, Grinvald A (1995) Optical imaging of the primary visual cortex in behaving monkey. Isr J Med Sci 31:766.

Shtoyerman E, Arieli A, Slovin H, Vanzetta I, Grinvald A (2000) Longterm optical imaging and spectroscopy reveal mechanisms underlying the intrinsic signal and stability of cortical maps in V1 of behaving monkeys. J Neurosci 20:8111-8121.

Toga AW, Cannestra AF, Black KL (1995) The temporal/spatial evolution of optical signals in human cortex. Cereb Cortex 5:561-565.

Tommerdahl M, Favorov O, Whitsel BL, Nakhle B, Gonchar YA (1993) Minicolumnar activation patterns in cat and monkey SI cortex. Cereb Cortex 3:399-411.

Tommerdahl M, Whitsel BL, Vierck CJ, Favorov O, Juliano S, Cooper B, Metz C, Nakhle B (1996a) Effects of spinal dorsal column transection on the response of monkey anterior parietal cortex to repetitive skin stimulation. Cereb Cortex 6:131-155.

Tommerdahl M, Delemos KA, Vierck CJ, Favorov OV, Whitsel BL (1996b) Anterior parietal cortical response to tactile and skin-heating stimuli applied to the same skin site. J Neurophysiol 75:2662-2670.

Tommerdahl M, Delemos KA, Favorov OV, Metz CB, Vierck CJ, Whitsel BL (1998) Response of anterior parietal cortex to different modes of same-site skin stimulation. J Neurophysiol 80:3272-3283.

Tommerdahl M, Delemos KA, Whitsel BL, Favorov OV, Metz CB (1999a) Response of anterior parietal cortex to cutaneous flutter versus vibration. J Neurophysiol 82:16-33.

Tommerdahl M, Whitsel BL, Favorov OV, Metz CB, O'Quinn BL (1999b) Responses of contralateral SI and SII in cat to same-site cutaneous flutter versus vibration. J Neurophysiol 82:1982-1992.

Ts'o DY, Frostig RD, Lieke EE, Grinvald A (1990) Functional organization of primate visual cortex revealed by high resolution optical imaging. Science 249:417-420.

Vanzetta I, Grinvald A (1999) Cortical activity-dependent oxidative metabolism revealed by direct oxygen tension measurements; implications for functional brain imaging. Science 286:1555-1558.

Vanzetta I, Grinvald A (2000) Evidence and lack of evidence for the initial dip in the anesthetized rat: implications for human functional brain imaging. NeuroImage 13:959-967.

Vnek N, Ramsden BM, Hung CP, Goldman-Rakic PS, Roe AW (1999) Optical imaging of functional domains in the cortex of the awake and behaving monkey. Proc Natl Acad Sci USA 96:4057-4060.

Wood CC, Spencer DD, Allison T, McCarthy G, Williamson PD, Goff WR (1988) Localization of human sensorimotor cortex during surgery by cortical surface recording of somatosensory evoked potentials J Neurosurg 68:99-111.

Yacoub E, Le TH, Ugurbil K, Hu XP (1999) Further evaluation of the initial negative response in functional magnetic resonance imaging. Magn Reson Med 41:436-441. 